\title{
Isothermal Combustor Prediffuser \& Fuel Injector Feed Arm Design Optimization Using the Prometheus Design System
}

\author{
Xu Zhang, David J.J. Toal* \\ Andy J. Keane \\ University of Southampton \\ Southampton, UK, SO16 7QF \\ Frederic Witham \\ Jonathan Gregory \\ Rolls-Royce plc. \\ Bristol, UK, BS34 7QE \\ Murthy Ravikanti, Emmanuel Aurifeille \\ Simon Stow, Mark Rogers, Marco Zedda \\ Rolls-Royce plc. \\ Derby, UK, DE24 8BJ
}

The Prometheus combustor design system aims to reduce the complexity of evaluating combustor designs by automatically defining pre-processing, simulation and postprocessing tasks based on the automatic identification of combustor features within the CAD environment. This system enables best practice to be codified and topological changes to a combustor's design to be more easily considered within an automated design process. The following paper presents the Prometheus combustor design system and its application to the multi-objective isothermal optimization of a combustor prediffuser and the multi-fidelity isothermal optimization of a fuel injector feed arm in combination with a surrogate modelling strategy accelerated via a high performance graphical processing unit.

\author{
Nomenclature \\ 1 Vector of ones of length $n$ \\ $C$ Multi-fidelity Kriging correlation matrix \\ $d$ Number of dimensions \\ d Difference vector \\ $E[I(\boldsymbol{x})] \quad$ Expected improvement \\ $n$ Number of sample points \\ $P$ Pressure \\ $p \quad$ Kriging smoothness parameter \\ $\boldsymbol{R}$ Correlation matrix \\ $\boldsymbol{r}$ Correlation vector \\ $s^{2} \quad$ Predicted error \\ $\boldsymbol{x}$ Vector of design variables \\ $\boldsymbol{Y}$ Combined vector of cheap and expensive data \\ $y$ Objective function value \\ $\boldsymbol{Z}$ Gaussian process \\ $\lambda$ Loss coefficient \\ $\theta$ Kriging rate of correlation decrease \\ $\phi$ Concentrated log-likelihood
}

*Corresponding author: djjt@ soton.ac.uk

\author{
$\hat{\mu} \quad$ Kriging mean \\ $\hat{\sigma}^{2} \quad$ Kriging variance \\ $\rho \quad$ Multi-fidelity scaling factor \\ Subscript \\ c Cheap or low-fidelity function evaluation \\ $d$ Difference between low and high fidelity \\ $e$ Expensive or high-fidelity function evaluation \\ $s$ Static pressure \\ $t$ Total pressure
}

\section{Introduction}

A typical engineering design process can involve a considerable number of design changes each of which may be followed by an assessment of the impact of those changes on multiple performance metrics. Such processes can involve multiple instances of geometry generation along with multidisciplinary simulations at varying operating conditions. The manual effort required in such design studies can be considerably reduced by employing modern design automation and optimization techniques and there are, of course, numerous examples of such techniques being applied throughout the literature. Aerofoil sections [1,2], compressor blades [3], wings [4], aircraft [5], combustors [6-8] and whole engines $[9,10]$, for example, have all been the subject of automated design optimizations in recent years. However, the majority of engineering design optimization examples within the literature include a fundamental limitation which can limit the benefits that such automation can bring to real world problems.

As the following paper is concerned with the design of a gas turbine combustor let us consider such a system as an example. Consider a rich burn combustion module with a set of dilution ports whose diameters are to be defined in order to improve the temperature profile at the combustor's exit. The diameters can be parameterized within a CAD pack- 
age and linked to a design table or similar. A script defining the meshing strategy can be defined and used repeatedly throughout the optimization as can scripts defining the CFD simulation and its post-processing. This optimization workflow could be used to investigate various combinations of dilution port diameters and even other design issues as long as the topology of the combustion module does not change.

To illustrate the impact of a relatively simple topological change on the above workflow let us also permit the number of dilution ports to vary. The generation of the geometry, in this instance, is not really an issue, an integer within a design table can be easily used to define the number of ports around the circumference of the combustor. However, assuming our script to define the meshing process includes commands to implement mesh refinement around the dilution ports to correctly capture flow behaviour, a change in the number of ports can result in, a refinement zone in the wrong location, a port with no refinement zone at all or redundant refinement zones. If the whole combustor module is being simulated the script defining the simulation may, in this instance, be retained as may the post-processing script, but this, of course, depends on the definition of the simulation and the performance metrics of interest. The extraction of dilution port mass flows during post-processing may necessitate an update in the script with every geometry change. Similarly if the combustor simulation is defined based on prescribed mass flow splits at each of the outlets the simulation boundary conditions may also have to be updated to reflect a change in the geometry.

The above simple example illustrates that while a fixed design optimization workflow can be repeatedly applied to sizing studies, modifications may be required as soon as the problem's topology is altered which may require considerable manual intervention thereby delaying the design process. Of course, even sizing problems can introduce problems within a workflow. The refinement zones applied around each of the dilution ports in the above example should have a mesh size directly linked to the size of the corresponding port. While not impossible to implement within a sizing study it does increase the complexity of the workflow.

The Prometheus combustor design system aims to facilitate the seamless implementation of topological changes within a design optimization workflow. The following paper presents this system and it's application, along with an advanced surrogate modelling system employing a graphical processing unit (GPU), to the multi-objective optimization of a combustor prediffuser and the multi-fidelity optimization of a combustor fuel injector feed arm.

The paper commences with an overview of the Prometheus system including its ability to cope with topological changes. This is followed by a brief introduction to surrogate modelling, specifically the Kriging and multi-fidelity Kriging techniques employed within the case studies. Also presented at this point is the acceleration of the construction and evaluation of a Kriging model using a graphical processing unit. This is an important advancement given the size of the surrogate models constructed in the second study. Details of the combustor geometry and associated meshing, simu-

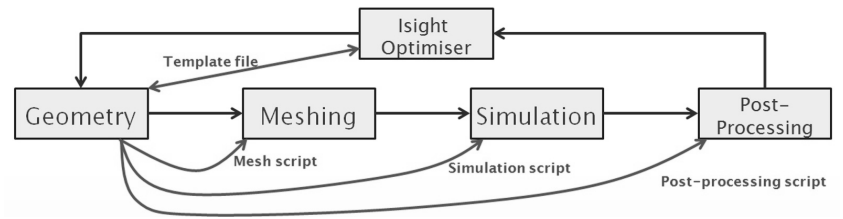

Fig. 1. A graphical representation of the geometry centric Prometheus optimization workflow [11]

lation and post-processing procedures used in the presented case studies are then followed by the results of both studies.

\section{An Overview Of The Prometheus Design System}

First presented by Zhang et al. [11], the Prometheus combustor design system completely removes fixed or template meshing, simulation and post-processing scripts from a design optimization workflow. Instead a 'geometry centric' approach (see Figure 1) is employed whereby bespoke scripts are generated automatically by the CAD system based on a feature identification process. This approach has a number of benefits:

1. The design optimisation workflow becomes considerably less sensitive to changes in the topology of the underlying geometry. This gives the designer more freedom to consider a greater range of changes within a design study and reduces the amount of rework required to modify the workflow.

2. Simulation as well as pre- and post-processing best practice can be captured and codified within a single program thereby offering a simple mechanism for new techniques to be rolled out and ensuring that approved methods are followed in all studies.

As illustrated in Figure 1, Prometheus' geometry centric approach employs a single XML input file which is passed to the CAD package, in this case Siemens NX. This file contains the names of the CAD files to be modified and interrogated along with a list of the required scripts to be output and information to override the defaults within the program as required. This can include anything from the full path of the output fluid volume file to adjustments to the rules defining mesh refinement zones in order to easily produce multiple levels of simulation fidelity for use in a multi-fidelity optimization

At the core of the design system sits the Prometheus plugin for Siemens NX. Developed using the Siemens NX Open $\mathrm{C}$ and $\mathrm{C}++$ application programming interface (API), this program parses the defined XML input file and executes each operation in turn. Figure 2 illustrates the general order in which operations are carried out by Prometheus.

As part of a combustor design study the process typically commences with the geometry of the combustor module. Part files defining either parametric or non-parametric features are loaded by Prometheus into memory. At this point Prometheus will make any changes to the geometry requested via the input file. Such changes can be via the mod- 

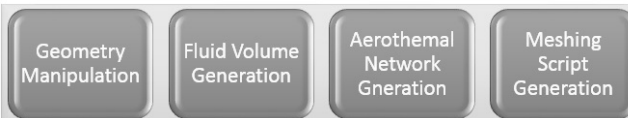

CFD \& Post-
Processing

Gneration

Generation

Processing

Fig. 2. A graphical representation of the operations carried out by the Prometheus CAD plug-in [11]

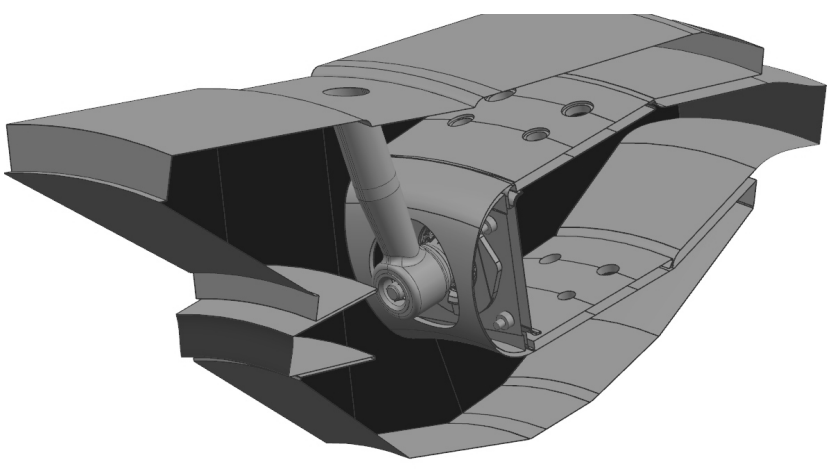

Fig. 3. An illustration of a Prometheus generated fluid volume [11]

ification of existing parametric expressions within a part file or through adjustments to the faces of a solid body via NX's synchronous modelling capability. Expressions can also be evaluated at this point allowing distances, areas and volumes to be calculated and used as constraints within an optimization.

With the combustor geometry modified as desired the process then proceeds to the generation of a CFD fluid volume. This process is key to the generation of the subsequent meshing, simulation and post-processing scripts with the information extracted during the generation of the fluid volume used to inform the creation of these scripts. The input file defines the name of the combustor assembly along with the names of parts defining key combustor components such as the prediffuser, outer and inner casing, the combustor itself and the injector. This definition of CAD parts allows different geometries to be combined together to create a combustor. The outer casing from one combustor and injector from another can be scaled via their parameterisations and joined together into a single CFD fluid volume and simulated.

The fluid volume extraction process is built around a series of geometry querying functions which are used to query faces of the solids within the parts and include/exclude these faces to/from the CFD volume and/or chain together series of faces which meet a common criteria. Using this approach the main faces defining the combustor fluid volume can be identified and extracted. With the main geometry extracted individual features within this geometry can be identified using combinations of the same set of geometry rules. In this way dilution ports, swirler passages, vanes and studs, to name but

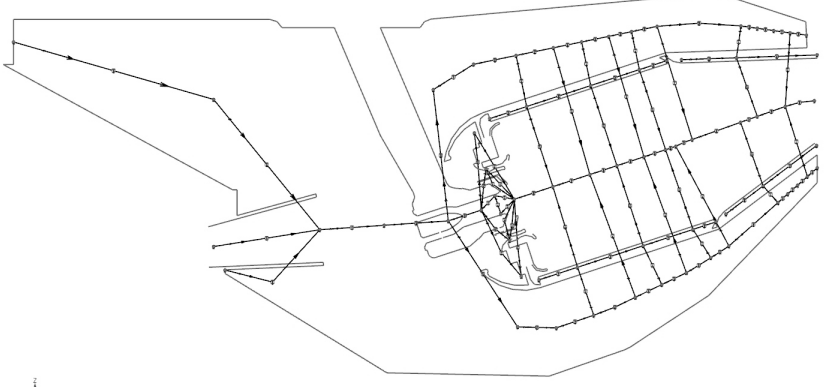

Fig. 4. An illustration of a Prometheus generated aerothermal network model [11]

a few, can be identified and directly extracted to the fluid volume and have critical dimensions calculated for use in the creation of any subsequent scripts. Figure 3 illustrates a CFD fluid volume generated by Prometheus from a series of separate CAD files.

With the faces of the fluid volume defined those features necessary to define a CFD simulation but which are not present within the original geometry are generated by the program. This includes the creation of the periodic boundaries, inlet and outlet planes as well as any planes required for post-processing the flowfield within the annuli, swirler passages, prediffuser, dilution ports and combustor exit. All sheet bodies extracted from the solid part files and created are given unique names which are then mapped through to the meshing, simulation and post-processing scripts.

With a fluid volume defined the next step, as illustrated in Figure 2, is for Prometheus to generate an aerothermal network model. This model can be used solely to assess the quality of a combustor design during preliminary design or to help define the boundary conditions of a CFD simulation. In either case the information extracted during the fluid volume generation is used to define annuli and prediffuser centrelines via a rolling ball, dilution port positions and sizes, duct diameters etc. within a Flownet $[6,7,12]$ aerothermal network model.

The manual generation of such a network is a laborious process and can become a bottleneck in the combustor preliminary design process. Prometheus' automated creation of such networks from a CAD assembly not only offers a solution to this issue but enables best practice regarding the creation of such networks to be captured in one place, updated if required and used by all design engineers.

With a network model created, run and then parsed by Prometheus the system moves on to the generation of an appropriate meshing script. As noted above the fluid volume construction process first identifies and then extracts information regarding important features within the geometry. Examples of this include the identification of the dilution ports and the subsequent calculation of their location, size, type and defining faces. Other examples include the identification of fuel swirler passages and vanes along with their minimum passage height. Other, more general, information regarding the scale of the module is also extracted, for exam- 


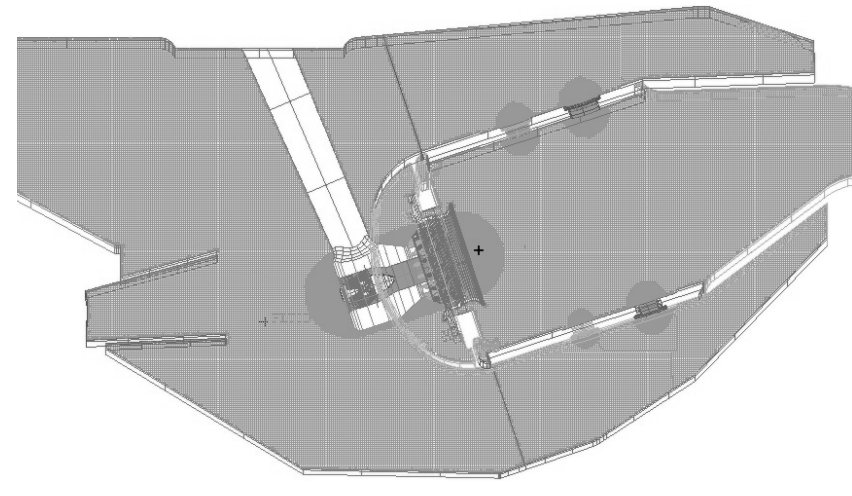

Fig. 5. An example mesh generated using an ICEM script created automatically by Prometheus [11]

ple, the combustor exit height.

General information is used to define, for example, the global mesh size and the location of the mesh volume within the meshing script created by Prometheus. Feature information, however, is used to define mesh refinement zones and local mesh sizes in accordance with the embedded meshing best practice. Taking a dilution port as an example, the diameter of the port along with an embedded rule on the minimum number of cells across the port is used to define the cell size within a refinement zone centered on the port which is large enough to encompass the port and any chutes present. As the diameter of the port varies the level of refinement will therefore adjust automatically to meet the embedded meshing rule or, if the port grows large enough, no mesh refinement will be specified at all. The same process is used to define mesh refinement zones around the fuel spray nozzle with the passage height now becoming the driving dimension.

Figure 5 illustrates a cross-section through the mesh generated for the fluid volume illustrated in Figure 3. The mesh refinement zones around the dilution ports and the fuel spray nozzle are clearly observable. It should be noted, however, that in this instance the refinement zones are defined as spherical, or cylindrical zones with spherical ends. Prometheus offers two methods of defining mesh refinement within ICEM with the system also capable of defining a local mesh size on a prescribed face with a fixed number of elements of this size perpendicular to the face followed by a managed growth to the global mesh size. Such an approach is employed within the case study presented in Section 5 and can be observed in Figure 17.

As noted above the input XML script permits some of the embedded default settings to be varied if desired and the definition of the meshing script is a perfect example of this. Both the defined minimum number of elements across the swirler passages and dilution ports can be varied via the XML thereby permitting multiple levels of fidelity to be defined with ease.

The final script created by the Prometheus system defines the simulation and post-processing operations within the proprietary CFD package PRECISE or commercial package ANSYS Fluent. The boundary conditions for the CFD simulation including mass flows, velocities and pressures, are defined based on the results of the aerothermal network simulation generated, run and subsequently parsed by Prometheus. In particular, the results of this simulation define the prediffuser inlet conditions and mass flow splits at the outlets along with mass flows leaving the annuli via effusion through the combustor walls and the mass flow entering the combustor through the walls. Walls, inlets and post-processing planes are all defined based on the fluid volume generated by Prometheus along with an ignition zone and fuel injector location.

In addition to the basic simulation setup Prometheus also defines additional simulation parameters such as the number of iterations prior to and after ignition, the turbulence model, the discretization scheme etc. Post-processing commands are also automatically included to calculate NOx, soot and other quantities of interest on the defined planes. Each of these parameters is based on the simulation best practice defined within Prometheus but can be adjusted if necessary via the XML input script.

With a fluid volume, successful aerothermal network simulation and meshing, CFD simulation and postprocessing scripts all automatically generated within the NX CAD environment an optimization workflow then only has to call the relevant software in batch and parse the results.

\section{Prometheus in the Presence of Topological Changes}

Having presented an overview of the Prometheus system let us now consider an example of its operation in the presence of both topological and sizing changes to a combustor design. Figure 6(a) illustrates a close-up of the fluid volume illustrated in Figure 3. In particular this figure shows both the fuel spray nozzle along with the size and topology of the dilution ports on both the inner and outer walls of the combustor. Figure 6(b) however, illustrates the fluid volume generated by Prometheus after the combustor geometry has undergone a number of changes. The original fuel spray nozzle has been replaced by a completely different design and the studs connecting the heatshield to the meterpanel have been removed. In addition to this the diameters of a number of dilution ports have been altered significantly.

As noted above, once a fluid volume has been generated Prometheus then generates a Flownet aerothermal network. The network illustrated in Figure 7 is that resulting from the geometric changes to the combustor presented above. While topologically similar to the network in Figure 4 there is a notable difference. The original combustor design had four rows of ports where the diameter of each port was identical across a row. As illustrated in Figure 6(b) the ports along the second row on the inner wall are no longer identical, one port is much larger than the other. Because of this change the topology of the network illustrated in Figure 4 is not quite correct. In order to accurately model the flow in this region the network should have two separate flow paths which represent the separate port diameters. As can be observed in the circled region of Figure 7 this topological change to the network has been successfully created by Prometheus. In this instance as the ports have been extracted the system has 


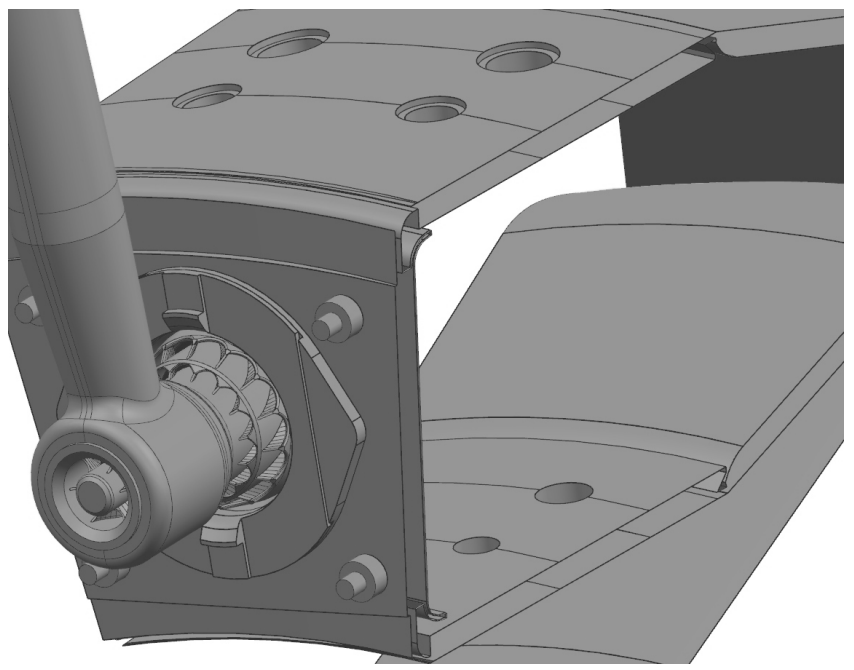

(a)

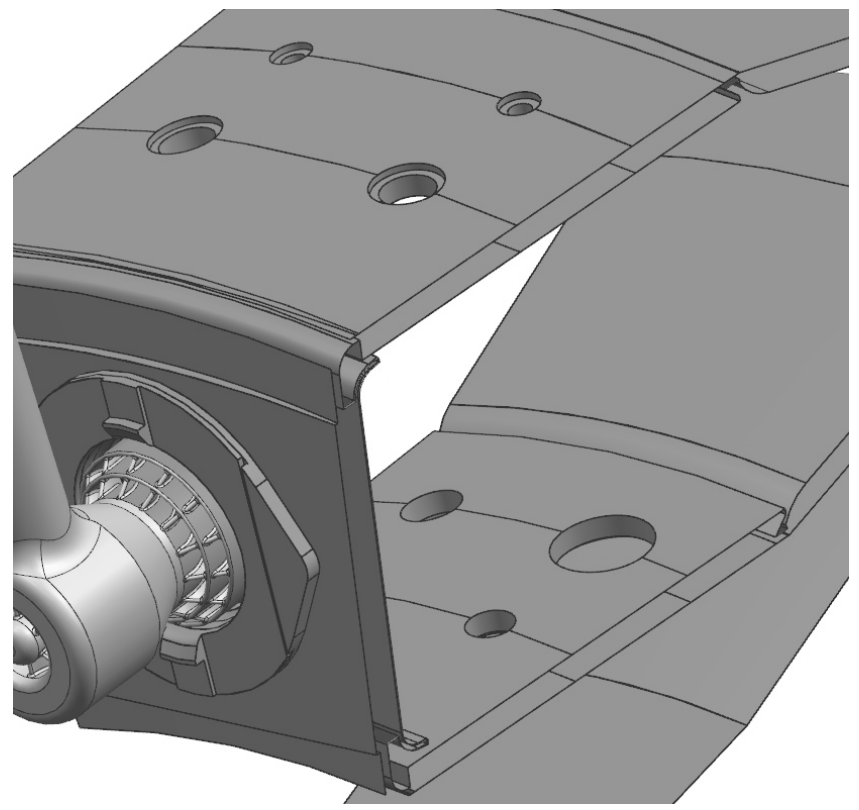

(b)

Fig. 6. A closeup view of the air swirler and combustor in the original (a) and modified geometry (b) [11]

recognised that there are two different port diameters and the network should reflect this.

As previously noted the dilution ports are a key driver in the definition of an automated meshing strategy. In particular the diameter of the port and the embedded, or user defined, required minimum number of cells across the port diameter are used to define a mesh refinement strategy in this region. Adjustments to the port diameters illustrated in Figure 6(b) should therefore be automatically reflected in the final mesh.

Figure 8 illustrates cross-sections through the mesh around the secondary row of dilution ports generated from the ICEM meshing script automatically created by Prometheus for the modified combustor geometry. Both of the outer ports in the secondary row were reduced in size quite considerably compared to the original design. The

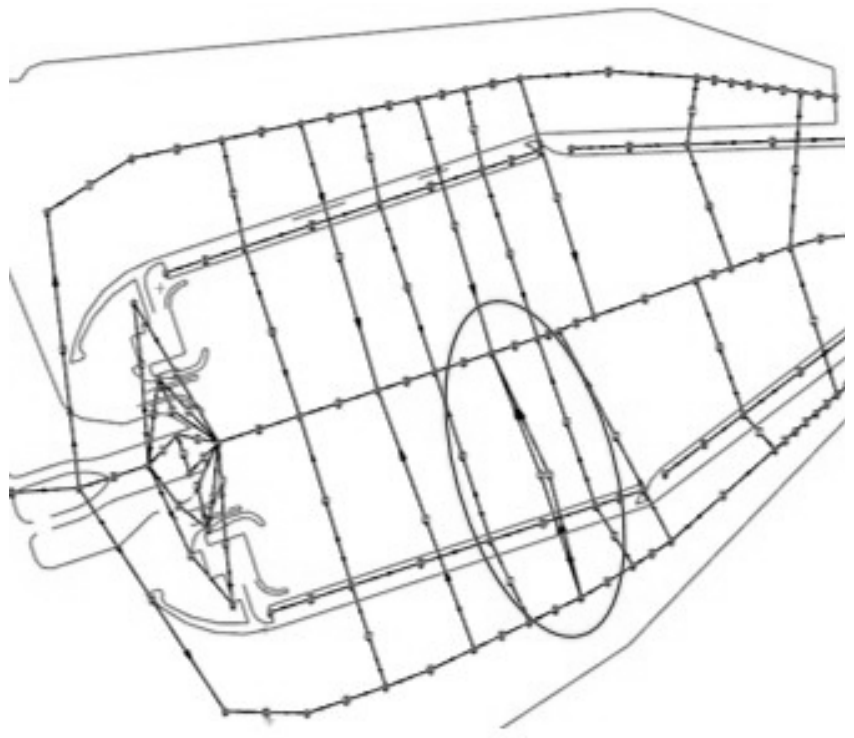

Fig. 7. A closeup view of the aerothermal network model resulting from the modified combustor design

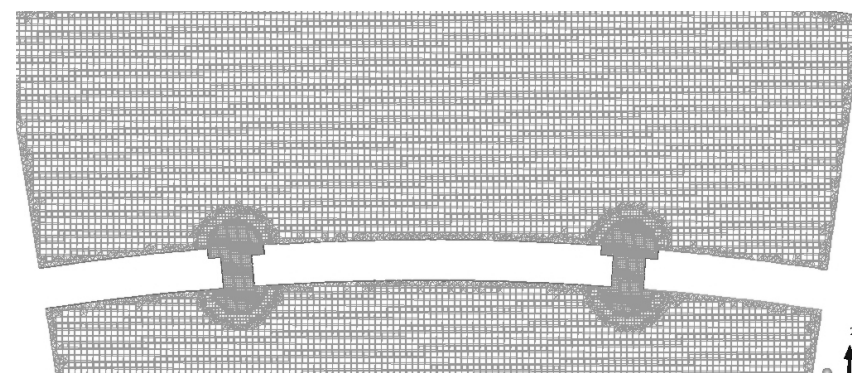

(a)

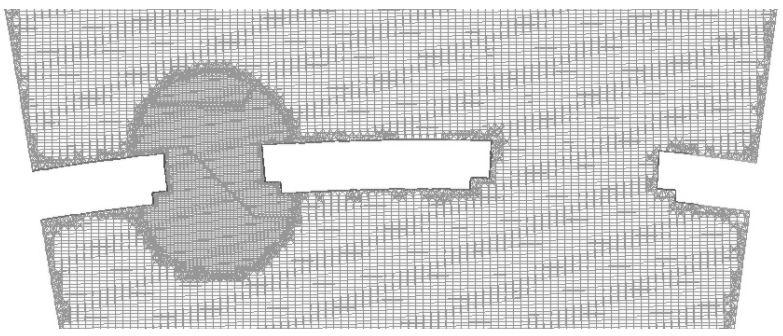

(b)

Fig. 8. Cross-section of the mesh through the outer (a) and inner (d) secondary row of dilution ports resulting from the Prometheus generated meshing script for the modified combustor.

mesh illustrated in Figure 8(a) demonstrates that Prometheus has not only automatically resized the refinement zone to match the new diameter but has introduced a secondary refinement zone with a slightly larger element size. This reflects Prometheus' attempt to respect it's embedded meshing rules regarding the way in which refinement zones can grow out to the global mesh size. Only factors of $\frac{1}{2^{i}}$ of the global 


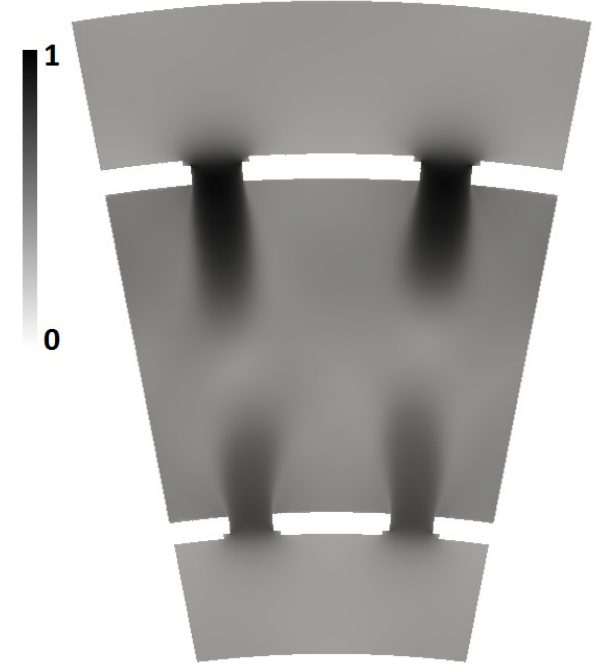

Fig. 9. Contours of normalized velocity magnitude through the secondary row of dilution ports for the original design

mesh size are permitted, where $i$ in this instance defines the number of refinement levels. In order to meet the minimum number of elements across the small ports a scale factor of $\frac{1}{4}$ is required. The embedded meshing rules state that the mesh should not increase from a factor of $\frac{1}{4}$ of the global mesh to the global mesh size directly so a second, slightly larger, refinement zone of $\frac{1}{2}$ the global mesh size is also centered on the ports. This change in mesh size can be clearly observed in Figure 8(a).

Figure $8(\mathrm{~b})$ reflects the mesh refinement rules working in a slightly different manner. Here the port on the right has seen its diameter increase over that of the baseline. Upon comparing the global mesh size to the diameter of the port the meshing script generation routine has determined that no refinement zone is required in order to meet the embedded minimum number of cells. The final mesh, therefore does not have a refinement zone placed around this port.

Of course, the changes in the geometry reflected in the aerothermal network and meshing script should be reflected in the CFD simulation of the new geometry. Figure 9 presents a contour plot of velocity magnitude on a plane through the inner and outer secondary row of dilution ports for the original design. Figure 10, however, illustrates the velocity contours on the same plane but for the modified design. Comparing the two contour plots one can clearly observe an acceleration of the flow through the outer ports and annulus and a slight increase in the velocity of the flow in the inner annulus and ports. The mixture of inner port diameters also considerably reduces the symmetry of the flow within the combustor.

The above example could, of course, be considered as rather arbitrary in its nature. However, it serves as an excellent demonstration of the Prometheus system's ability to cope with both topological and sizing changes to a combustor

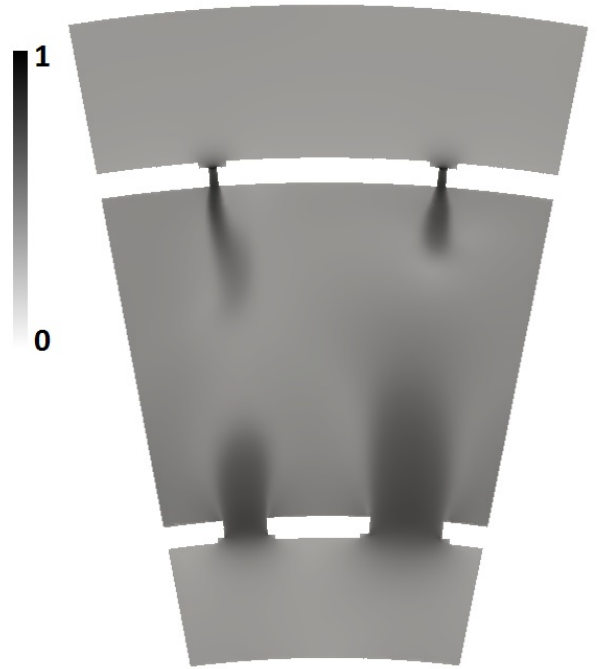

Fig. 10. Contours of normalized velocity magnitude through the secondary row of dilution ports for the modified design

geometry with necessary changes to the aerothermal network and mesh being made automatically and reflecting in a final CFD simulation. With these capabilities demonstrated the remainder of this paper will focus on the application of the system to two isothermal combustor design studies namely the multi-objective optimization of the combustor prediffuser and multi-fidelity optimization of the fuel injector feed arm both for reduced annuli pressure loss.

\section{Surrogate Modelling}

The Prometheus system, as outlined above, is designed to automatically generate various pre- and post-processing and simulation scripts automatically from combustor geometry files. The system therefore enables a workflow defining the automatic evaluation of combustor design changes to be generated reasonably quickly. This, however, does nothing to help the fact that both the mesh generation and the CFD simulation can take a considerable amount of time to complete and are therefore a considerable bottle neck. Within a design optimization process the Prometheus system must therefore be employed in conjunction with an efficient optimization system which reduces the number of function evaluations and therefore the number of CFD simulations that need to be performed.

Surrogate modelling techniques, otherwise known as metamodelling techniques or response surface modelling, offer a way in which the total number of function evaluations for an optimization can be reduced. This is achieved by evaluating the true objective function at a series of sampling points defined by, for example, a Latin Hypercube design of experiments, and then using this data to construct a mathematical model which represents the variation in the objective function with variations in the magnitude of the 
design parameters. This surrogate model can then be optimized instead of the true objective function thereby reducing the number of computational simulations required compared to a direct search such as a genetic algorithm. The optimal point or points identified upon searching the surrogate can then be assessed using the true objective function, the surrogate model updated and the cycle repeated until convergence or a specified number of function evaluations have been exhausted. The interested reader can find further details on general surrogate modelling approaches within the literature [13-15].

Both of the case studies presented within this paper employ surrogate modelling in order to reduce the number of expensive CFD simulations of the combustor. Before considering these case studies let us first present the surrogate modelling techniques employed.

\subsection{Kriging}

Kriging is one of the most popular forms of constructing a surrogate model due to its flexibility and useful prediction of the error in the model which can be used to derive a probability of improvement and an expected improvement over the current best design.

Popularized by Sacks et al. [16], Kriging assumes that if two design points, $\boldsymbol{x}_{i}$ and $\boldsymbol{x}_{j}$, are close together then the corresponding objective function values, $y\left(\boldsymbol{x}_{i}\right)$ and $y\left(\boldsymbol{x}_{j}\right)$ will be similar. This assumption is modelled by assuming that the correlation between two sets of random variables, $Y\left(\boldsymbol{x}_{i}\right)$ and $Y\left(\boldsymbol{x}_{j}\right)$, is described by,

$$
\boldsymbol{R}_{i j}=\operatorname{Corr}\left[Y\left(\boldsymbol{x}_{i}\right), Y\left(\boldsymbol{x}_{j}\right)\right]=\exp \left(-\sum_{l=1}^{d} 10^{\boldsymbol{\theta}_{l}}\left\|\boldsymbol{x}_{i_{l}}-\boldsymbol{x}_{j_{l}}\right\|^{\boldsymbol{p}_{l}}\right)
$$

where $\boldsymbol{\theta}_{l}$ and $\boldsymbol{p}_{l}$ represent the so-called hyperparameters of the $l^{\text {th }}$ variable and, respectively, determine the rate of correlation decrease and the degree of smoothness in each direction.

The construction of a Kriging model involves the definition of an appropriate set of these hyperparameters given the observed data set. This is achieved via an optimization of the concentrated log-likelihood function of Jones [17],

$$
\phi=-\frac{n}{2} \ln \left(\hat{\sigma}^{2}\right)-\frac{1}{2} \ln (|\boldsymbol{R}|)
$$

where the optimal variance and mean are,

$$
\hat{\sigma}^{2}=\frac{1}{n}(\boldsymbol{y}-\mathbf{1} \hat{\mu})^{T} \boldsymbol{R}^{-1}(\boldsymbol{y}-\mathbf{1} \hat{\mu})
$$

and

$$
\hat{\mu}=\frac{\mathbf{1}^{T} \boldsymbol{R}^{-1} \boldsymbol{y}}{\mathbf{1}^{T} \boldsymbol{R}^{-1} \mathbf{1}}
$$

The concentrated log-likelihood function is dependent only on the correlation matrix, $\boldsymbol{R}$, whose elements are defined by Equation 1, and hence on the model's hyperparameters. More information on the derivation of the likelihood function can be found in Jones' original papers [17, 18].

The likelihood function which is optimized in order to define an optimal set of hyperparameters can be multi-modal which necessitates the use of a global optimization algorithm such as, for example, a genetic algorithm. These techniques can require a significant number of function evaluations which due to the repeated factorization of the correlation matrix can lead to the hyperparameter optimization becoming rather expensive. As will be demonstrated later this is particularly the case when the model is constructed from a large number of sample points.

The literature contains a number of techniques to accelerate the convergence of the hyperparameter optimization. In the following paper the hybrid particle swarm algorithm of Toal et al. $[19,20]$ which utilizes an adjoint of the likelihood function within a local search is employed.

Given an optimized set of hyperparameters a prediction of the Kriging model at a new point, $\boldsymbol{x}^{*}$, can be calculated by first determining a vector of correlations between the unknown point and the sample points, $\boldsymbol{r}\left(\boldsymbol{x}^{*}\right)$. This can then be used in conjunction with the mean calculated using Equation 4 to form the predictor,

$$
y\left(\boldsymbol{x}^{*}\right)=\hat{\mu}+\boldsymbol{r}^{T} \boldsymbol{R}^{-1}(\boldsymbol{y}-\mathbf{1} \hat{\mu}) .
$$

As noted above, Kriging provides a useful estimate of the error in the prediction,

$$
s^{2}\left(\boldsymbol{x}^{*}\right)=\hat{\boldsymbol{\sigma}}^{2}\left[1-\boldsymbol{r}^{T} \boldsymbol{R}^{-1} \boldsymbol{r}\right],
$$

which can be used to calculate the probability of improvement, or as is also used in this paper, the expected improvement [18] over the current minimum, $y_{\min }$,

$$
\begin{aligned}
E\left[I\left(\boldsymbol{x}^{*}\right)\right] & =\frac{\left(y_{\min }-y\left(\boldsymbol{x}^{*}\right)\right)}{2}\left[1+\operatorname{erf}\left(\frac{y_{\min }-y\left(\boldsymbol{x}^{*}\right)}{s \sqrt{2}}\right)\right] \\
& +\frac{s}{\sqrt{2 \pi}} \exp \left[\frac{-\left(y_{\min }-y\left(\boldsymbol{x}^{*}\right)\right)^{2}}{2 s^{2}}\right] .
\end{aligned}
$$

\subsection{Multi-Fidelity Kriging}

All surrogate modelling techniques, Kriging included, are dependent on the amount of data used in their construction. With a large amount of data the resulting surrogate model will generally be an accurate representation of the true response and the subsequent surrogate based optimization will converge relatively quickly. However, if only a relatively small sampling plan can be afforded, as may be the case when an expensive simulation is employed, the resulting surrogate model may be inaccurate. While this may not prevent a global optimum being obtained, as long as the general 


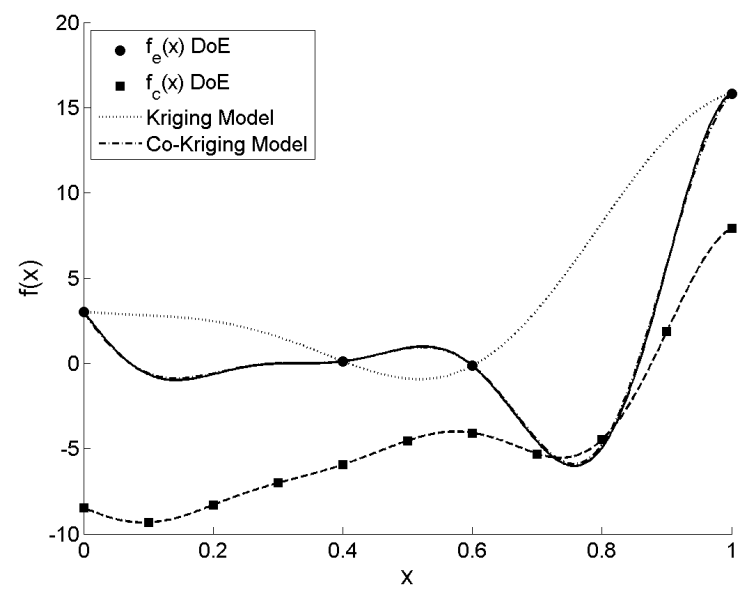

Fig. 11. Co-Kriging example using the Forrester function [21]

trends in the response are correct, it may slow convergence of the optimization.

Multi-fidelity surrogate modelling techniques aim to improve the global accuracy of the model by including a mixture of high and low fidelity data. As long as the low fidelity data is well correlated with the high fidelity data [22] the low fidelity data can be used to better define the trend in the response in regions of the design space where there is little or no high fidelity data.

Figure 11, recreated from Forrester et al. [21], is an excellent example of the advantages of a multi-fidelity approach. Here the single fidelity surrogate model is constructed through four data points evaluated using a high fidelity, or expensive function, $f_{e}(x)$. The resulting Kriging model interpolates the data points but looks very little like the true function it is attempting to represent. A multi-fidelity Kriging model, otherwise referred to as a Co-Kriging model, constructed using the same four high fidelity data points and an additional eleven data points sampled from the low fidelity, or cheap, function $f_{c}(x)$, is almost indistinguishable from the true expensive function and will locate the global optimum with a single update.

As demonstrated above, such an increase in surrogate model accuracy can considerably accelerate the convergence of the resulting optimization and reduce overall simulation effort. The literature contains a number of examples of the effectiveness of this technique on problems including airfoil design [23-25], the creation of aerodynamic models [25-29], compressor blade design [3] and even whole engine optimization [10].

Multi-fidelity Kriging is an extension of standard Kriging developed by Kennedy and O'Hagan [30] to capture multiple levels of simulation fidelity. Using their approach the high fidelity response is then approximated by multiplying the low fidelity response by a scaling factor, $\rho$, and a Gaussian process representing the difference between the high and low fidelity data,

$$
Z_{e}(\boldsymbol{x})=\rho Z_{c}(\boldsymbol{x})+Z_{d}(\boldsymbol{x}) .
$$

If $\boldsymbol{X}_{e}$ and $\boldsymbol{X}_{c}$ represent the expensive and cheap data respectively, then the covariance matrix $\boldsymbol{C}$ is,

$$
\boldsymbol{C}=\left(\begin{array}{cc}
\sigma_{c}^{2} \boldsymbol{R}_{c}\left(\boldsymbol{X}_{c}, \boldsymbol{X}_{c}\right) & \rho \sigma_{c}^{2} \boldsymbol{R}_{c}\left(\boldsymbol{X}_{c}, \boldsymbol{X}_{e}\right) \\
\rho \sigma_{c}^{2} \boldsymbol{R}_{c}\left(\boldsymbol{X}_{e}, \boldsymbol{X}_{c}\right) & \rho^{2} \sigma_{c}^{2} \boldsymbol{R}_{c}\left(\boldsymbol{X}_{e}, \boldsymbol{X}_{e}\right)+\sigma_{d}^{2} \boldsymbol{R}_{d}\left(\boldsymbol{X}_{e}, \boldsymbol{X}_{e}\right)
\end{array}\right)
$$

where the correlations are of the same form as Equation 1.

As can be observed from Equation 9 there are now two sets of hyperparameters which must be determined. One set for the model of the low fidelity data and another for the model representing the difference between the scaled low fidelity data and the high fidelity data. In addition to this the scaling parameter, $\rho$ is also unknown and must be determined.

The construction of such a multi-fidelity model commences with the creation of a single fidelity Kriging model of the low fidelity dataset. Using the methodology briefly outlined above, the hyperparameters of the model, $\boldsymbol{\theta}_{c}$ and $\boldsymbol{p}_{c}$ are optimized using the standard maximum likelihood approach.

With the low fidelity model defined the scaling parameter and the second set of hyperparameters are determined by replacing the $\boldsymbol{y}$ in the above equations with a vector of differences, $\boldsymbol{d}$ between the low fidelity model and the high fidelity data points where,

$$
\boldsymbol{d}=\boldsymbol{y}_{e}-\rho \boldsymbol{y}_{c}\left(\boldsymbol{X}_{e}\right)
$$

The construction of the difference model proceeds as before but with the scaling parameter, $\rho$ also considered as a variable within the maximum likelihood optimization.

With the hyperparmeters for both models defined the multi-fidelity prediction of the high fidelity response is given by,

$$
y_{e}\left(\boldsymbol{x}^{*}\right)=\hat{\mu}+\boldsymbol{c}^{T} \boldsymbol{C}^{-1}(\boldsymbol{y}-\mathbf{1} \hat{\mu}),
$$

where the mean is now,

$$
\hat{\mu}=\frac{1^{T} C^{-1} Y}{1^{T} C^{-1} 1},
$$

and where $\boldsymbol{Y}$ is a combination of the known low and high fidelity responses, $\boldsymbol{Y}^{T}=\left[\boldsymbol{y}_{c}^{T}, \boldsymbol{y}_{e}^{T}\right]$. Further details on the derivation of this predictor and the likelihood optimization can be found in Kennedy and O'Hagan [30]. As per single fidelity Kriging an estimate of the error in the predictor can be calculated and the expected improvement over the current best design calculated as before [21]. 


\subsection{GPU Accelerated Surrogate Model Construction}

As noted above a large sampling plan and the use of a global optimization algorithm with a large number of function evaluations can cause the Kriging likelihood optimization to be rather expensive to perform. This could be considered as even more of an issue with a multi-fidelity model due to the propensity for there to be a large sampling plan defining the low fidelity surrogate due to the relative cheapness of the objective function.

While techniques such as the hybridized particle swarm employed here can reduce the number of function evaluations by making the optimization more efficient they do nothing to reduce the cost of a single evaluation of the loglikelihood function, Equation 2. Similarly, when a Kriging or Co-Kriging model is constructed from a large sampling plan the evaluation of the prediction, error estimate, probability of improvement and expected improvement can also become expensive due to the size of the matrix-matrix and matrixvector multiplications involved. As shall be demonstrated in the second case study considered within this paper both of these issues when combined can introduce something of a bottleneck in the optimization process by first delaying the construction of the surrogates and then the search of those surrogates.

A novel solution to this problem, considered within the current paper, is the application of a graphical processing unit (GPU) to accelerate the construction of a Kriging model. With their parallel processing prowess, GPUs have become increasingly used to help accelerate the solution of a variety of problems in a number fields such as engineering, physics and finance. Even modestly priced personal computers now come equipped with some form of GPU which, although typically used for playing games or other graphics intensive activities such as image processing or computer aided design, could easily be harnessed to accelerate traditional CPU based activities.

Let us therefore consider the application of a GPU to the construction and evaluation of a Kriging model. In this instance both the CPU and GPU implementations of the Kriging functions are developed using Matlab and its inbuilt GPU toolbox. It should also be noted at this point that all of the surrogate modelling and optimization processes presented within this paper, including the GPU acceleration are implemented within the proprietary Rolls-Royce optimization suite OPTIMATv2 [9, 19, 20,31,32], itself written in Matlab.

Figure 12 presents a comparison of the cost associated with the calculation of the log-likelihood function as both the problem dimensionality and the number of sample points, $n$, increases when the function is evaluated using a desktop CPU operating in single and multi-threaded modes and two different GPUs. The first GPU considered, the Nvidia Quadro 2000M is a low-end laptop graphics card and is representative of what might be found in even the most basic system. The second card considered, the Tesla K20C is representative of a cutting edge high performance graphics card especially designed for parallel computing. Comparing both cards gives an indication of the magnitude of the performance gains which might be attained with different levels of investment.

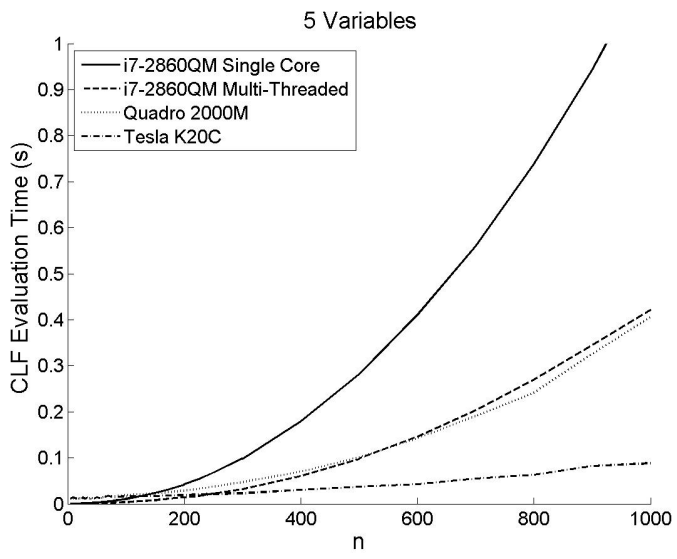

(a)



(b)

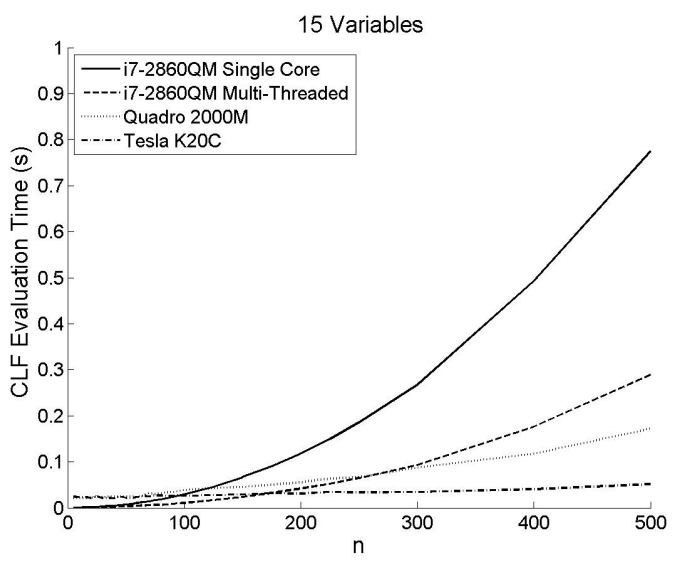

(c)

Fig. 12. Comparison of concentrated log-likelihood evaluation costs for 5 (a), 10 (b) and 15 dimensional problems when using a i7-2860 CPU and Quadro 2000M and Tesla K20C GPUs

It is immediately clear from Figure 12 that for even moderate problems there is a significant reduction in the evaluation time of the likelihood function when evaluated using a GPU. An evaluation of the likelihood for a five dimensional 


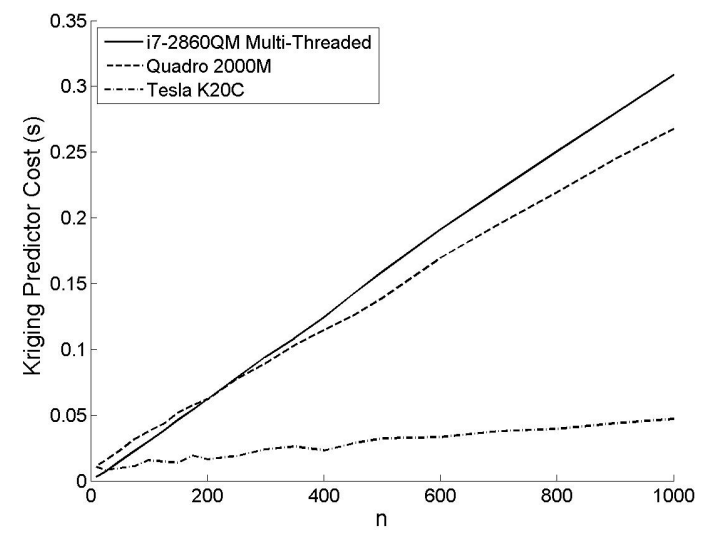

Fig. 13. Comparison of Kriging predictor evaluation costs for a 5 dimensional problem when using an i7-2860 CPU and Quadro 2000M and Tesla K20C GPUs and evaluating 1000 points in parallel

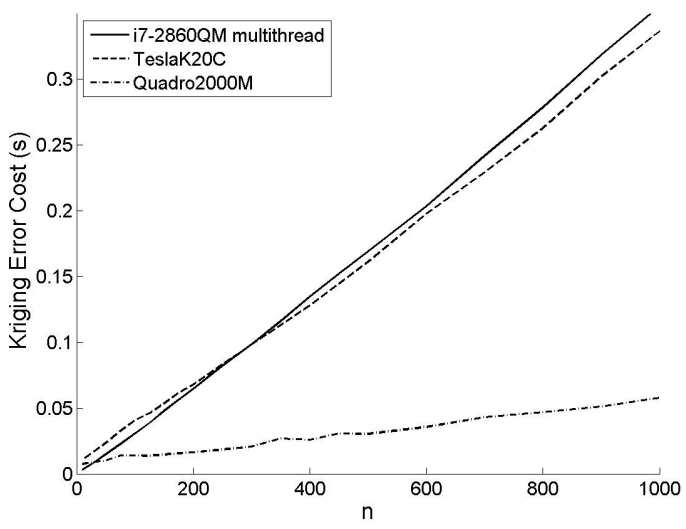

Fig. 14. Comparison of Kriging error evaluation costs for a 5 dimensional problem when using an i7-2860 CPU and Quadro 2000M and Tesla K20C GPUs and evaluating 1000 points in parallel

Kriging model with 1000 sample points, for example, can be evaluated on the Tesla GPU in $25 \%$ of the time it takes the CPU. Of course the results also illustrate that there is an overhead associated with using a GPU to evaluate the likelihood function in cases where the sampling plan typically contains under 200 points. What is striking from Figure 12 is that even the relatively cheap Quadro $2000 \mathrm{M}$ offers a performance advantage over the CPU when the models have more than 5 dimensions.

The most expensive aspect of evaluating the likelihood function is the factorisation of the correlation matrix which is of $\mathrm{O}\left(n^{3}\right)$ and does not scale particularly well when parallelized. The evaluation of the Kriging predictor and error functions, Equations 5 and 6 respectively, however, mainly involve vector-matrix multiplications which should benefit much more from the parallelization offered by a GPU.

Figures 13 and 14 illustrate just this, with the high performance Tesla card outperforming the multi-threaded CPU even at very modest sampling sizes when evaluating the pre-

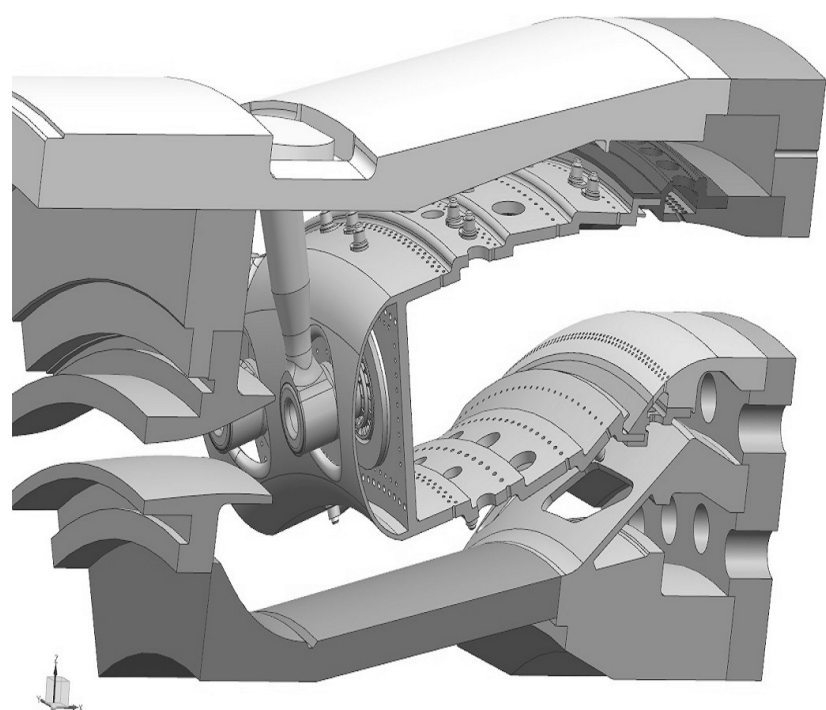

Fig. 15. Double sector CAD model of the baseline combustor experimental rig including injectors and prediffuser

dictor and error estimate at 1000 unknown points in parallel. While not performing as well as the Tesla card the cheaper Quadro GPU does outperform the CPU when the size of the sampling plan defining the surrogate model becomes large. Once again there is an overhead associated with the GPU operations but this occurs at a much lower sample size.

While Figures 12, 13 and 14, consider only Kriging, it should be observed that the same accelerations can be obtained with a multi-fidelity Kriging model. As described above the process of constructing a multi-fidelity Kriging model is closely related to that of ordinary Kriging. The construction of the low fidelity model is, in fact, the construction of an ordinary Kriging model whereas the construction of a high fidelity model involves very little additional effort over the standard likelihood calculation apart from the calculation of the vector of differences. However, it should be pointed out that the construction of the difference model may be faster on a CPU than on a GPU if the sampling plan is small and the relative performance of the GPU is impacted by the associated overhead. Of course the use of GPU or CPU can be determined dynamically prior to any optimization commencing.

\section{Combustor Case Study Description}

With both the Prometheus system and the surrogate modelling and optimization tools described let us now consider the design problem which forms the basis of the following case studies.

The basis for both design case studies is the combustor module illustrated in Figure 15. At this stage it should be pointed out that this geometry represents a perspex rig constructed at Loughborough University, similar to that pre- 
sented by Cha et al. [33] and is representative of a combustion module for a large civil engine.

The case study geometry does not include a set of tiles although the thickness of the combustor casing has been defined so that the size of the combustor flametube is similar to that of a combustor with tiles. Both geometries contain dilution ports on both the inner and outer walls of the combustor but to represent the effusion present on a real combustor design a large number of additional small holes have been drilled into the combustor wall. In addition to this, the perspex rig geometry has a number of studs on the annuli walls representative of those used to hold tiles in place. An accurate representation of the platform for the nozzle guide vane (NGV) is included as is an accurate representation of the combustor rear inner casing (CRIC). Finally, although not clearly visible in Figure 15 a front mounting pin is also included in the rig. The geometry presented in Figure 15 is therefore a topologically very different to that illustrated in Figure 3.

As noted above the first task of the Prometheus system is always the generation of the fluid volume from the combustor CAD assembly with which it is presented. The following case studies are no different and Prometheus is required to generate such a volume from the CAD parts presented in Figure 15. Prometheus was initially developed as a tool to support and accelerate the preliminary design process while the case study geometry is more representative of the detailed design stage. As such a number of additions are included within the system to cope with the identification and extraction of the tile studs as well as the NGV platform and CRIC. With these functions included Prometheus can generate both a single and double sector fluid volume of the case study geometry, as illustrated in Figure 16. Figure 16 also illustrates the generation of a series of post-processing planes upstream of the first row of dilution ports in both the inner and outer annuli as well as at the exit of the prediffuser and at the start of the NGV platform.

With a fluid volume generated Prometheus then generates a script to mesh the volume within ICEM CFD. Figure 17 illustrates a cross-section through the center of the mesh of the fluid volume presented in Figure 16(a). As with the similar combustor geometry all of the dilution ports and the holes representing effusion have been identified automatically during the fluid volume generation process and mesh refinement zones have been defined in these locations based on the hole diameter and the global mesh size. Similarly, refinement within the air swirler passages has been automatically defined based on the passage height. The refinement of both the holes in the combustor casing and within the swirler can be clearly observed in Figure 17.

The isothermal CFD simulations carried out as part of both designs studies are performed using ANSYS Fluent. As this is an experimental rig the inlet profile and exit flow splits are assumed to be known apriori and fixed. There is therefore no need in this instance to generate an aerothermal network from which to populate the CFD boundary conditions. The realizable k- $\varepsilon$ turbulence model is used throughout with an initial 200 iterations run using a first order discretization



(a)

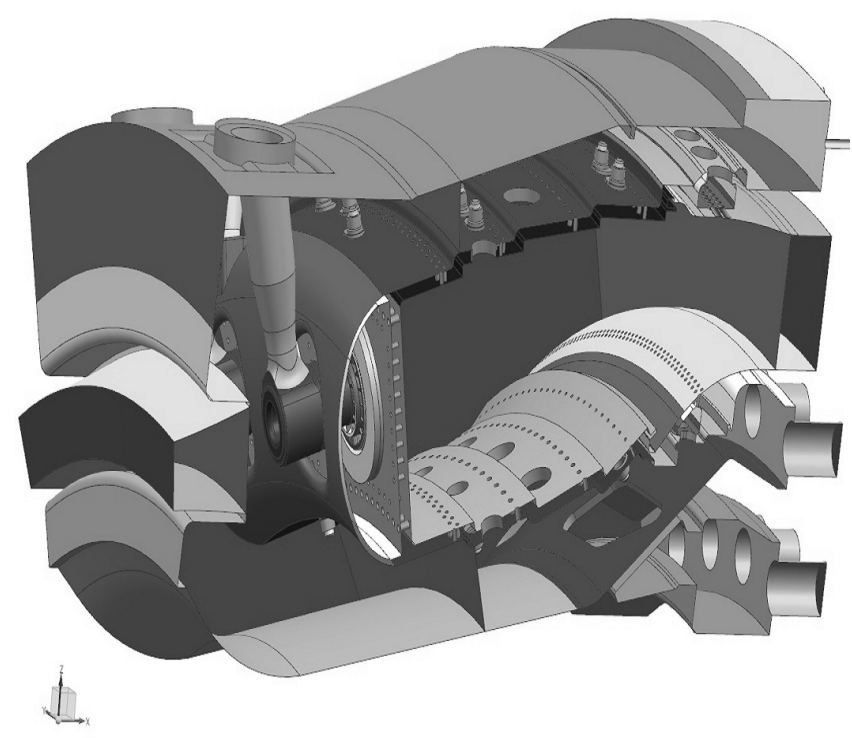

(b)

Fig. 16. Single (a) and double (b) sector fluid volumes generated by Prometheus including post-processing planes

scheme followed by a further 3000 iterations using a second order scheme. Both the CFD simulations and mesh generation are performed on a 16 core computer with $256 \mathrm{~Gb}$ of ram with a single sector simulation requiring approximately 29 hours of runtime in total and a double sector simulation requiring 104 hours. As can be seen in Table 1 the majority of the runtime for both cases is due to the CFD simulation.

The post-processing for every simulation is performed 


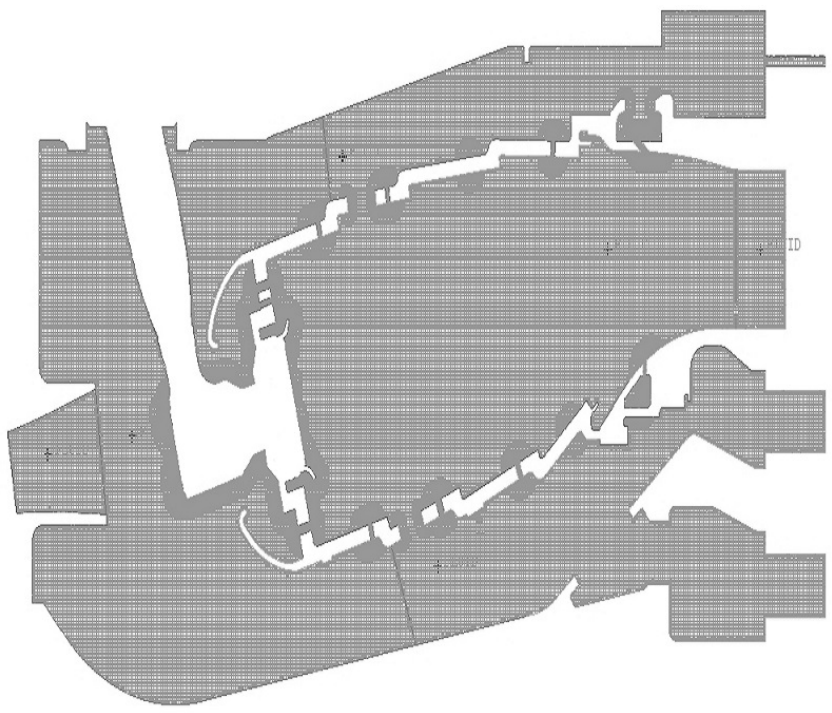

Fig. 17. Cross-section through the baseline computational mesh illustrating refinement zones around the injector, dilution ports and liner holes

Table 1. Typical meshing and simulation runtimes

\begin{tabular}{lll}
\hline No. of Sectors & 1 & 2 \\
Meshing time (16 cores) (hrs) & 4 & 16 \\
No. mesh elements & $\approx 31.4 \mathrm{~m}$ & $\approx 60.9 \mathrm{~m}$ \\
CFD runtime (16 cores) (hrs) & 25 & 88 \\
Total runtime (hrs) & 29 & 104 \\
\hline
\end{tabular}

within Fluent. At the conclusion of a simulation the mass averaged total and static pressures are reported at four postprocessing planes defined within the fluid volume and at the domain inlet. This data is then used to calculate the loss coefficient, $\lambda$, at each post-processing plane where,

$$
\lambda_{\text {plane }}=\frac{P_{t_{\text {inlet }}}-P_{t_{\text {plane }}}}{P_{t_{\text {inlet }}}-P_{S_{\text {inlet }}}}
$$

where $P_{t}$ and $P_{s}$ define total and static pressure respectively, the subscript "inlet" defines the domain inlet and the subscript "plane" defines a value at one of the four postprocessing planes. In both of the subsequent design case studies the losses between the domain inlet and inner and/or outer annuli post-processing planes are the subject of the optimizations.

\section{Multi-Objective Prediffuser Optimization}

The first case study considered within this paper involves the multi-objective optimization of the combustor prediffuser with respect to the pressure losses at the postprocessing plane in the inner and outer annulus. In this case

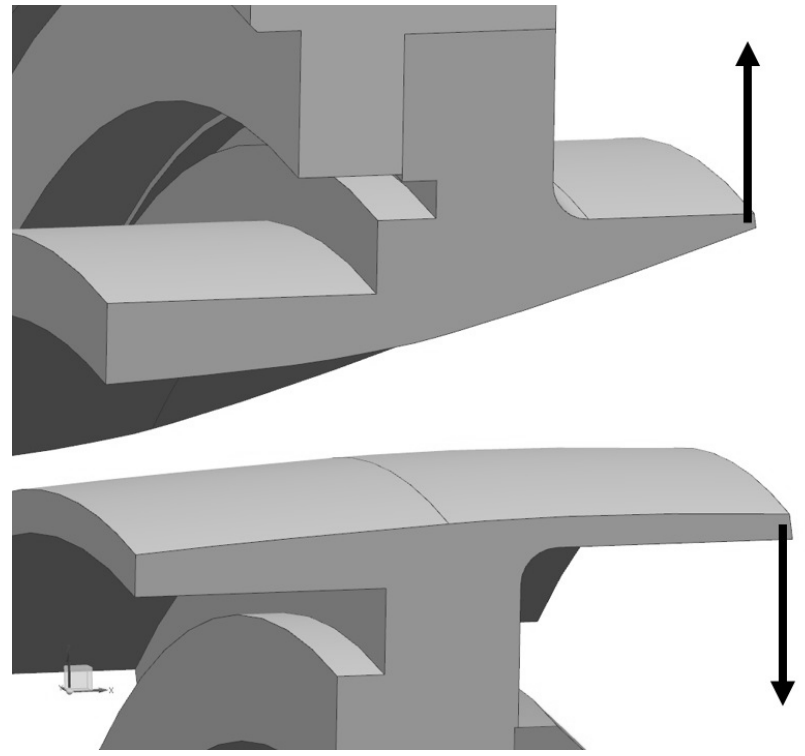

Fig. 18. Graphical illustration of the prediffuser design parameters

Prometheus is used to adjust the prediffuser geometry, generate a meshing and a simulation script. An Isight workflow employing the OPTIMATv2 surrogate modelling and optimization plugin is then used to control the design parameters considered during the optimization as well as the generation of any surrogate models.

Both the inner and outer walls of the prediffuser are parameterized using an arc, the end points of which can be moved in a radial direction. Figure 18 illustrates the independent movement of the prediffuser walls. The walls are moved completely independently which permits a widening and contraction of the exit along with a redirection of the flow exiting the prediffuser. The meshing and simulation process outlined above in Section 5 is employed throughout the study and only single sector simulations of the module are performed. Each simulation, including meshing, therefore takes approximately 29 hours to perform.

As already noted, the overall aim of this optimization is to improve the pressure losses at the entrance to both of the annuli. A weighted combination of these losses could be employed in such an optimization, however, this would not give the full picture of the trade-off between the two losses and differences in their scales might bias the optimization. Instead a multi-objective optimization study is performed in order to obtain a Pareto front of improvement in the inner annulus pressure loss versus improvement in the outer. Naturally such an optimization may improve design performance at the flow conditions considered at the expense of performance at off-design conditions. To prevent this from occurring two design points for the combustor will be considered. The optimization is primarily concerned with improving performance at cruise conditions but this will be constrained by a requirement for there to be no loss in performance at maximum take-off (MTO). For each combustor design considered during the optimization two CFD simulations will therefore be performed, one at MTO and a second at cruise with the 


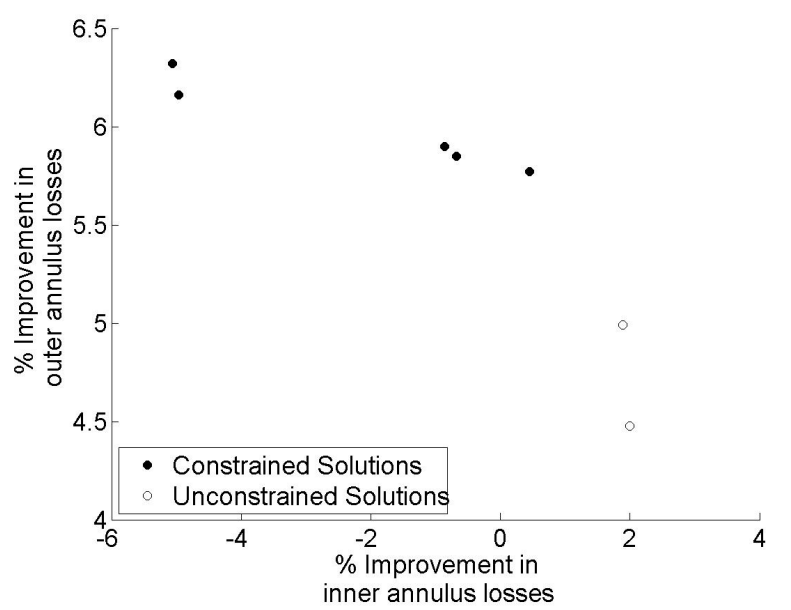

Fig. 19. Pareto front of inner and outer annuli pressure losses with those designs constrained by performance at MTO highlighted

pressure loss used to define constraints and objective functions respectively. The optimization considered within this case study can therefore be defined as,

$$
\begin{array}{ll}
\text { Minimize : } & \lambda_{\mathrm{I}_{\mathrm{cruise}}} \& \quad \lambda_{\mathrm{O}_{\text {cruise }}} \\
\text { Subject to }: & \lambda_{\mathrm{I}_{\mathrm{MTO}}} \leq \lambda_{\text {baseline }} \mathrm{I}_{\mathrm{MTO}} \\
& \lambda_{\mathrm{O}_{\mathrm{MTO}}} \leq \lambda_{\text {baseline }} \mathrm{O}_{\mathrm{MTO}} \\
& \boldsymbol{x}_{l} \leq \boldsymbol{x} \leq \boldsymbol{x}_{u}
\end{array}
$$

where subscripts I and $\mathrm{O}$ define the inner and outer annuli

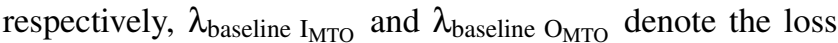
coefficients for the baseline design at MTO, $\boldsymbol{x}$ defines the design variables and the subscripts $u$ and $l$ denote the upper and lower bounds for the design variables.

The optimization commences with an 11 point design of experiments consisting of the baseline prediffuser design and a 10 point space filling sampling plan. The loss coefficients at cruise and MTO for both annuli are then used to construct a series of Kriging surrogate models. The Kriging predictors are then employed to search for a set of four update points along the Pareto front of improvement in outer versus improvement in inner annuli losses at cruise using NSGA II [34]. The two surrogate models of predicted MTO losses are employed as constraints during the search. A total of four sets of four updates are evaluated, which along with the initial sampling plan means that a total of 27 different prediffuser designs have been investigated during the optimization.

The results of this optimization are presented in Figures 19 and 20. Figure 19 demonstrates the Pareto front obtained from the optimization while Figures 20(a) and 20(b) display the location of the Pareto optimal points within the design space along with surrogate models of the improvement in inner and outer pressure losses respectively. To illustrate the impact of the constraint on MTO performance those designs which result in a reduction in performance at MTO are presented as filled circles while those for which no reduction in

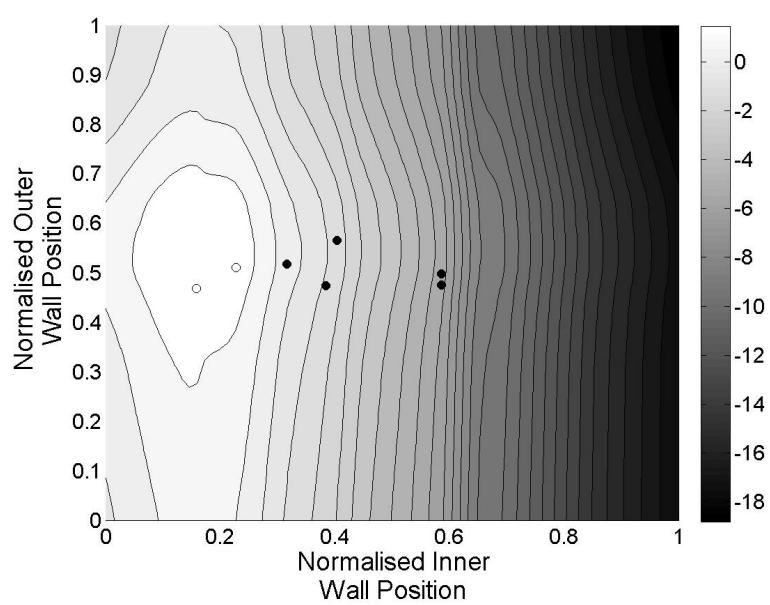

(a)

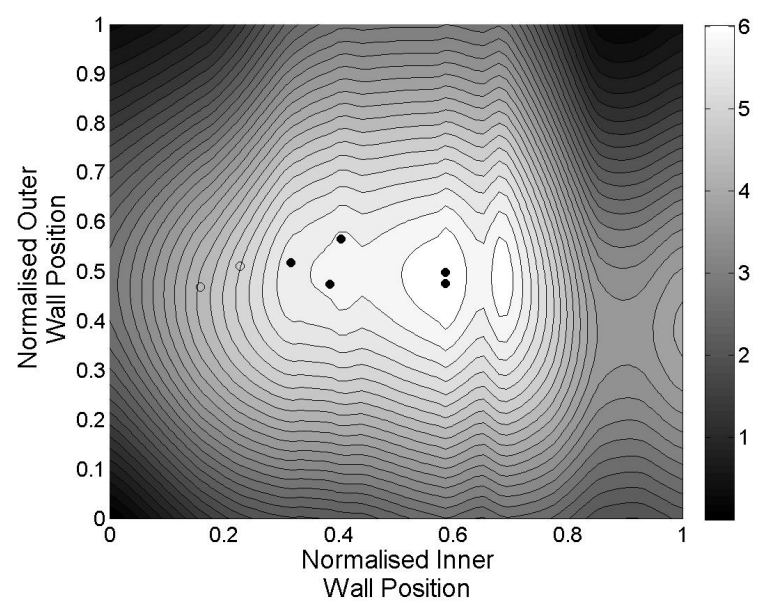

(b)

Fig. 20. Surrogate models of inner (a) and outer (b) annuli pressure losses with the constrained Pareto front highlighted

MTO performance is observed are presented as empty circles. It should be observed at this point that the baseline prediffuser design occurs at $[0,0]$ in Figure 20.

The Pareto front of Figure 19 demonstrates that an improvement of $2 \%$ in the inner annulus pressure loss is achievable and an improvement of over $6.25 \%$ is achievable for the outer annulus. However, the Pareto front demonstrates a clear trade-off between inner and outer annulus performance as the prediffuser design is altered. This is further reinforced by the surrogate model predictions of improvement in inner and outer losses in Figures 20(a) and 20(b). These surrogates indicate that the regions of maximum improvement for both annuli are completely different. The inner annulus loss is lowest when the the outer wall is moved outwards and the inner wall moves inwards a relatively small amount. The outer pressure loss, however, is improved most when the inner wall is moved much further towards the centreline of the combustor.

The surrogate predictions of improvement in inner and outer loss coefficients also demonstrate that the performance 


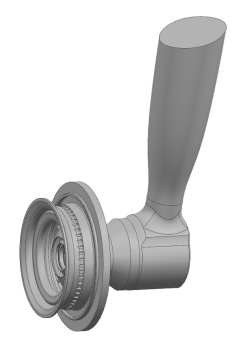

(a)

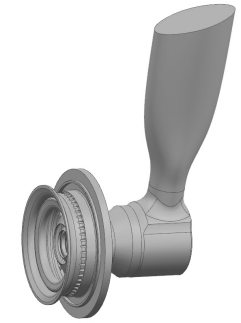

(b)

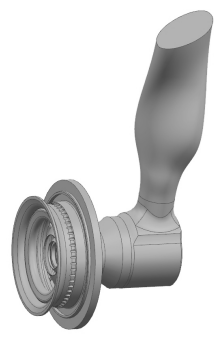

(c)
Fig. 21. Illustration of the baseline injector feed arm geometry (a), maximum major to minor ellipse axis ratio (b) and with three independently twisted sections

of the outer annulus is much less sensitive to the prediffuser geometry. Almost all combinations of prediffuser shape considered result in an improvement to the losses experienced by the outer annulus. The inner annulus, however, is much more sensitive to changes in the prediffuser shape with designs where the inner wall has moved further towards the centerline producing large increases in the loss coefficients. The surrogate model's prediction indicates a potential increase in the loss coefficient of over $18 \%$.

Figures 20(a) and 20(b) also indicate a level of symmetry in the response of annuli losses to changes in the outer wall position. The final Pareto front falls along the center of the design space when the normalised movement of the outer prediffuser wall is equal to approximately 0.5 . Moving the position of the outer wall further from this position results in an increase in the losses experienced by both annuli.

When the constraint on MTO performance is considered the number of feasible designs in the Pareto front is considerably reduced. While the outer annulus pressure loss can be improved relatively easily at cruise the performance at MTO is much more sensitive to changes in the prediffuser shape which considerably curtails the level of improvement at cruise that can be realised. After taking the MTO constraint into account a maximum improvement of approximately $5 \%$ can be obtained for the outer annulus with an improvement of approximately $2 \%$ in the inner annulus performance.

\section{Multi-Fidelity Fuel Injector Feed Arm Optimization 7.1 Problem Description}

Having considered the optimization of the combustor prediffuser shape for improvement in both inner and outer annuli pressure losses let us now consider the shape optimization of the combustor's fuel feed arm.

The baseline fuel feed arm, illustrated in Figure 21(a), is defined by a series of circular cross-sections along a common centreline. In order to optimize the feed arm shape these circular sections have been replaced by a set of three ellipses for which the ratio of the minor to major axis and offset of the major axis from the main combustor axis can be modified. When these parameters are varied simultaneously for the three sections a feed arm similar to that illustrated in Figure 21(b) is obtained. In this instance the feed arm can move from a fully circular design, as per the baseline, to one with an oval cross-section along its length. The major axis of each cross-section is permitted to vary between \pm 45 from the centreline.

Combining changes in the three cross-sections together results in a two variable parameterization and therefore a two variable optimization. The feed arm cross-sections can, however, also be adjusted independently resulting in a total of six design parameters giving the type of twisted feed arm illustrated in Figure 21(c).

Unlike the prediffuser optimization, the optimization of the fuel feed arm will be performed using double sector simulations and will be used to demonstrate the application of the Prometheus system within a multi-fidelity design optimization. Moving forward we will therefore consider the single and multi-fidelity optimization of the feed arm when parameterized by both two and six variables. In both optimizations only the impact of the feed arm shape on the outer annulus pressure loss coefficient is considered due to the relatively small impact of the feed arm shape on any inner annulus loss. Naturally the outer annulus is affected by the feed arm shape most as it lies directly in its wake.

\subsection{D Optimization}

The two variable optimization aims to reduces the outer annulus loss by varying the ratio of the major to minor axis of the cross-sections defining the feed arm and the angle of the major axis relative to the main combustor axis. Due to the expense of the double sector simulations no constraints on MTO performance are considered in either feed arm optimization. The optimization can therefore be quite simply defined as,

$$
\begin{array}{ll}
\text { Minimize : } & \lambda_{\mathrm{O}_{\text {cruise }}} \\
\text { Subject to : } & \boldsymbol{x}_{l} \leq \boldsymbol{x} \leq \boldsymbol{x}_{u} .
\end{array}
$$

Unlike the optimization of the prediffuser shape two different optimization approaches will be considered here and compared. The first employs a basic surrogate modelling approach with the results of a 10 point design of experiments sampling plan used to construct a Kriging model. This model is then searched using a genetic algorithm to maximise the expected improvement in the outer annulus loss. The design which maximises the expected improvement is then evaluated using the double sector CFD simulation with the outer annulus loss coefficient then used to update the model. This cycle of surrogate model construction and update is performed a total of five times resulting in a total of 15 double sector CFD simulations.

The second approach employs a multi-fidelity strategy. In this case single and double sector simulations are combined within a multi-fidelity Kriging model. The same 10 point sampling plan used in the single fidelity optimization is used to define a set of 10 single sector CFD simulations. 


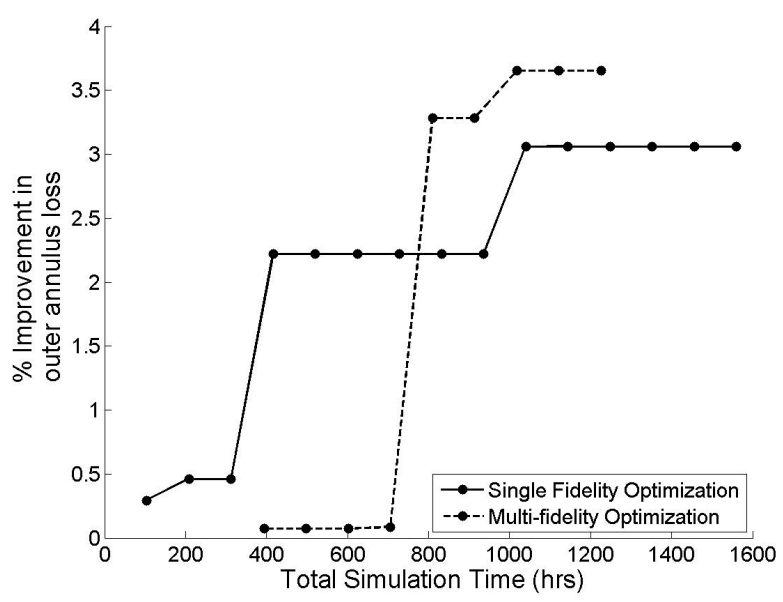

Fig. 22. Two variable feed arm search histories for single and multifidelity design optimizations as a function of total simulation time

An optimal four point space filling subset of this 10 point sampling plan is then used to define the double sector simulations. The loss coefficients resulting from these single and double sector simulations are then employed within a multifidelity Kriging model, as described above, with a set of five update simulations carried out using only double sector CFD simulations at the locations maximising the expected improvement. This multi-fidelity optimization therefore employs a total of 10 single sector CFD simulations and 9 double sector simulations resulting in a total compute time of approximately 1200 hours, compared to 1600 hours for the single fidelity optimization.

Figure 22 illustrates the search histories of both the single and multi-fidelity optimizations while Figure 23 illustrates the final feed arm designs. Note that the time for the first simulation of the multi-fidelity optimization has been shifted to account for the low fidelity single sector simulations which make up the low fidelity sampling plan.

Both the single and multi-fidelity design optimizations have resulted in similar designs with the ratio of the major to minor axis maximised in both cases and the angle of the major axes reasonably parallel to the axis of the combustor. However, as can be observed in Figure 22 the multi-fidelity optimization has located this design with considerably less simulation effort and has converged to a final solution before the sampling plan for the single fidelity optimization has been evaluated. After a total of 800 hours computation time the multi-fidelity simulation has achieved an improvement in the outer annulus loss of more than $3.25 \%$ whereas the single fidelity simulation has only achieved $2.25 \%$ and not improved upon the best design found in the design of experiments. The difference in performance observed between the final designs is due to the additional noise within the high fidelity design space which hampers convergence of the optimization to a cross-section setting angle of precisely $0^{\circ}$. This means that, in this instance, the multi-fidelity approach offers both a reduction in computational effort and an improvement

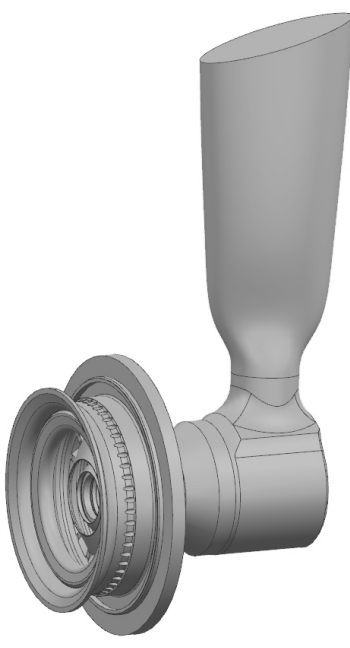

(a)

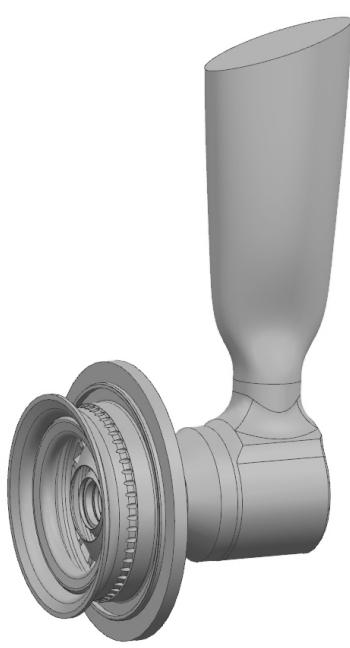

(b)
Fig. 23. Illustration of the injector stem geometry resulting from a two variable single (a) and multi-fidelity (b) design optimization, note that there is no real discernible difference in the geometries

in the final design.

\subsection{D Optimization}

Having considered a two dimensional multi-fidelity design optimization of the fuel feed arm let us now increase the flexibility of the parameterization and permit all six parameters to vary independently.

While the final designs resulting from the two variable optimization demonstrate an improvement in the losses observed in the outer annulus little attention was paid to the manufacturability of the final feed arm. Given the complex shapes which can potentially be created from the six variable parameterization (see Figure 21(c)) it becomes quite important to prevent infeasible designs during the optimization. To that end three geometry constraints are introduced as part of the following six variable optimization.

The first two constraints limit the degree of variation in twist along the length of the feed arm. That is they limit the difference between the first and second and second and third cross-section major axis angles. The setting angle of the second cross-section has to be within $36^{\circ}$ of the first crosssection while the angle of the third cross-section has to be within $18^{\circ}$ of the second cross-section. The difference in these constraints is due to the relative position of the defining cross-sections along the length of the feed arm with the second cross-section being half the distance from the third section as it is from the first.

The third and final geometric constraint concerns the fuel passage running along the center of the feed arm. As well as providing a structural mount for the air swirlers the feed arm acts like a heat shield and protects the fuel within the feed arm from coking etc. Any new feed arm design should not therefore encroach on the internal fuel passage or become so thin so as the heat from the combustion chamber 
negatively impacts on the fuel within the passage. To that end we make the assumption that the original feed arm met this design constraint and that the wetted surface of any new feed arm design should not come any closer to the fuel passage. This final constraint therefore ensures that the minimum wall thickness between the fuel passage and the wetted surface is greater than or equal to that of the original design.

As per the two variable optimization each combustor design will only be considered at cruise conditions with the outer annulus loss coefficient the subject of the optimization. The six variable design optimization considered here can therefore be defined as,

$\begin{aligned} \text { Minimize; } & \lambda_{\mathrm{O}_{\text {cruise }}} \\ \text { Subject to : } & t_{\min } \geq t_{\text {allowable }} \\ & \Delta \alpha_{1} \leq 36^{\circ} \\ & \Delta \alpha_{2} \leq 18^{\circ} \\ & x_{l} \leq \boldsymbol{x} \leq \boldsymbol{x}_{u}\end{aligned}$

where $t_{\min }$ defines the minimum calculated feed arm wall thickness, $t_{\text {allowable }}$ defines the minimum allowable distance and $\Delta \alpha$ defines absolute difference between the angle of each cross-section.

The surface of the feed arm is represented by a single b-spline surface within Siemens NX and, as illustrated in Figure 21(c), including twist within each cross-section and varying the level of ovality can result in a quite complex final surface. The variation in the minimum distance between this surface and the inner fuel passage can therefore be relatively non-stationary with regions of very little variation interspersed with regions of high variation. The accurate prediction of this constraint is therefore a key requirement for the optimization to progress and for feasible designs to be considered through an expensive CFD simulation. To improve the accuracy of the prediction of this and the other constraints a large 1000 point sampling plan was performed prior to carrying out any CFD simulations where only the geometry was varied and the constraints were evaluated. The surrogate model resulting from this sampling plan can then be used to seed the CFD simulations into regions which are known to be feasible a priori.

While this improves the ability of the optimization to consider feasible geometries it considerably increases the cost of the construction of the surrogate models representing the constraints as well as the cost of searching them. To reduce this cost the GPU accelerated Kriging model construction technique described previously in Section 4.3 is employed. This reduces the tuning time from approximately 30 minutes to approximately 10 minutes per surrogate model and reduces the time to construct and search all of the surrogates from 80 minutes to approximately 30 minutes, a considerable time saving of $62.5 \%$. While perhaps this appears insignificant compared to the cost of the CFD simulations used here, one should recall that the process of constructing and searching the surrogate models is performed after every batch of new combustor designs that are considered. The
Table 2. Accuracy of the Kriging models of the constraints determined via leave-one-out cross-validation

\begin{tabular}{cccc}
\hline & $\mathrm{r}^{2}$ & RMSE & MAE \\
\hline$\Delta \alpha_{1}$ & 1.000 & $3.86 \times 10^{-3}$ & $3.33 \times 10^{-2}$ \\
$\Delta \alpha_{2}$ & 1.000 & $3.69 \times 10^{-3}$ & $2.66 \times 10^{-2}$ \\
$t_{\min }$ & 0.998 & $3.25 \times 10^{-2}$ & $2.84 \times 10^{-1}$ \\
\hline
\end{tabular}

total wall time saved will therefore continue to grow as the optimization progresses.

Table 2 indicates the accuracy of the surrogate models of the constraints resulting from the 1000 point sampling plan. Naturally the surrogate models of the simpler angle ratios represent the response very accurately, both have a $\mathrm{r}^{2}$ correlation of approximately 1.0 and root mean square errors (RMSE) and maximum absolute errors (MAE) of $O\left(10^{-3}\right)$ and $O\left(10^{-2}\right)$ respectively. However, although it also employs a total of 1000 sample points in only six dimensions the surrogate model representing the wall thickness is not as accurate. While the $\mathrm{r}^{2}$ correlation is close to 1.0 the RMSE and MAE are both an order of magnitude larger than for the other two constraints.

As per the two variable feed arm optimization we will also use this case study to assess both a single and a multifidelity approach. Both optimizations will employ the same initial surrogate models of the three constraints with only the surrogate modelling approach for the objective function varying. The single fidelity optimization will consist of a 30 point sampling plan at which double sector CFD simulations are carried out. A Kriging model constructed from these simulations is then searched with the three constraint surrogate models used to predict the feasibility of the design. The multi-fidelity approach, however, simulates the same 30 point sampling plan using the cheaper single sector CFD simulation and augments these simulations with an optimal subset of 15 double sector CFD simulations. The single fidelity design of experiments therefore takes a total of approximately 3120 hours to compute while the multi-fidelity sampling plan takes approximately 2430 hours, a $22.1 \%$ time saving. In both optimization strategies updates to the surrogate model are considered in series with a total of six further double sector CFD simulations performed during each optimization.

Figure 24 illustrates the optimization search histories for both the single and multi-fidelity design optimizations. As with the two variable optimization the start of the multifidelity optimization history has been shifted to account for the cost incurred through the lower fidelity single sector CFD simulations. It should also be noted that only those designs which satisfy the three design constraints are plotted in Figure 24 and the fifth update to the multi-fidelity optimization failed to correctly generate a usable mesh and so the total time for this update is reduced to 16 hours. 


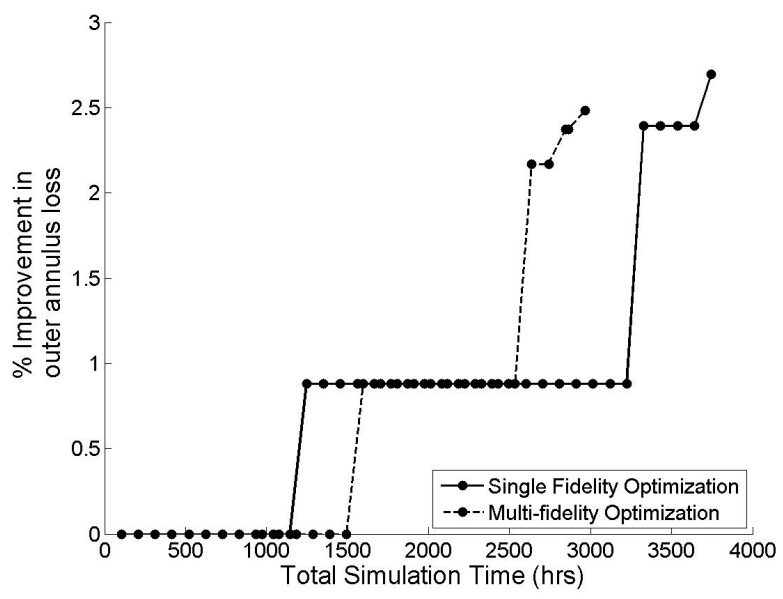

Fig. 24. Six variable feed arm search histories for single and multifidelity design optimizations as a function of total simulation time

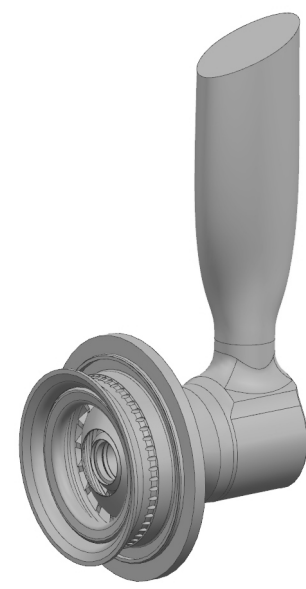

(a)

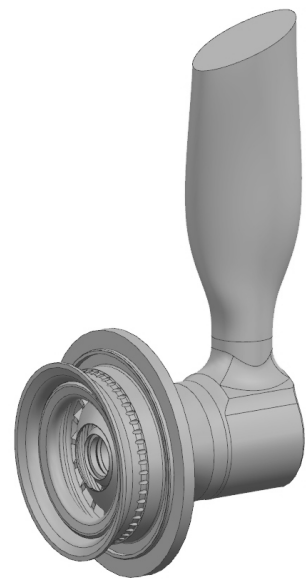

(b)
Fig. 25. Illustration of the injector stem geometry resulting from a six variable single (a) and multi-fidelity (b) design optimization

As with the two variable optimization the multi-fidelity approach demonstrates a clear acceleration in the convergence of the optimization. As noted above, the single fidelity optimization takes approximately 3120 hours to compute the design of experiments but within this time the multi-fidelity optimization has completed and been able to find a design offering a $2.5 \%$ improvement in outer annulus pressure loss. The single-fidelity optimization is only able to surpass the best design found by the multi-fidelity optimization after a approximately 3744 hours of simulation time whereas the multi-fidelity optimization has completed in approximately 2966 hours a $20 \%$ saving.

Figure 25 illustrates the final stem design resulting from both the single and multi-fidelity optimizations. Both optimizations have resulted in a very similar shape to the topmost section of the feed arm. The lengths of both the major and minor axes and the setting angles are very similar. In both cases the permitted level of ovality has been maximized and the feed arm has been twisted by approximately $20^{\circ}$ to the axis of the engine.

The lowermost section is also very similar in both cases. While the setting angle may be slightly different the level of ovality has been reduced here so as to prevent the constraint on the distance between the wetted surface and fuel passage being broken. With a relatively circular cross-section the angle has little impact producing a similar looking geometry in this region.

The major difference between the two feed arms occurs at the middle section. While the sections are very similar in terms of the level of ovality there is a considerable difference in their setting angles. The multi-fidelity optimization results in a section offset by approximately $13^{\circ}$ to the main engine axis while the single fidelity optimum is almost parallel.

While the final designs are broadly very similar, the single fidelity optimization has, in this instance, resulted in a slightly superior design (2.69\% compared to $2.48 \%$ improvement in pressure loss) but at the cost of an additional 778 hours (over 32 days) of compute time.

\section{Conclusions}

The following paper presents the Prometheus combustor design system, its general philosophy and the benefits that it offers within the design optimization of a combustor. The Prometheus system aims to move the creation of meshing, simulation and post-processing scripts to within the CAD environment where feature identification routines can easily take account of topological changes made as part of a design study. Changes, such as variations in the number of dilution ports which dramatically increase the complexity of a design workflow can be now be managed with relative ease.

The Prometheus system was applied to two isothermal combustor design optimization case studies. The system was shown to be able to extract the fluid volume for either a single or double sector simulation of the module, generate appropriate post-processing planes as well as meshing and CFD simulation scripts.

The first case study employed the Prometheus system within a constrained multi-objective design optimization of a combustor prediffuser where the inner and outer annuli pressure losses were to be improved subject to no increase in losses at MTO. The Pareto front resulting from this optimization demonstrated a trade-off between improving both the inner and outer annuli losses with reduced losses in one annulus coming at the expense of increased losses in the other. However, the optimization did demonstrate that an improvement of $2 \%$ and $5 \%$ in the respective inner and outer annuli losses could be realized simultaneously with no loss in performance at MTO.

The second case study employed the Prometheus system within the design optimization of a fuel injector feed arm. This case study was used to illustrate the ability of Prometheus to seamlessly generate multiple levels of simulation fidelity (in this case single and double sector CFD simulations), the general power of a multi-fidelity design approach and the ability of the GPU surrogate modelling rou- 
tines to speed up the construction of large models. The two variable design optimization demonstrated that a $3.75 \%$ improvement in the outer annulus loss could be obtained by modifying the cross-sectional shape of the feed arm from a circle to an oval and that a multi-fidelity approach was capable of achieving greater improvements in design performance over a shorter wall time.

This optimization, however, included no constraints on the feed arm geometry, in particular, no constraint was included to prevent the wetted surfaces of the feed arm coming too close to, or even intersecting, the internal fuel passage. Including this geometry constraint and increasing the flexibility of the feed arm parameterization to employ three independent cross-sections saw an improvement in the outer annulus pressure loss of $2.7 \%$ be obtained by a single fidelity optimization employing only expensive double sector CFD simulations. The multi-fidelity optimization, however, achieved a $2.5 \%$ improvement and a very similar final design but for a reduction of $20 \%$ in the total simulation time. The complexity of the geometry constraint to prevent intersections with the fuel passage necessitated the use of a surrogate model employing a large number of sample points which was demonstrated to be constructed and evaluated $62.5 \%$ faster when employing the GPU accelerated scheme presented within the current paper.

In conclusion, the Prometheus combustor design system has a number of features which enable it to considerably reduce the complexity in setting up an optimization workflow. It's ability to cope with topological changes means that scripts no longer need to be rewritten between different design studies or managed using complicated schemes. The design engineer is therefore free to concentrate on developing novel designs rather than the vagaries of implementing individual studies. The current article also demonstrates that the benefits offered by the Prometheus system are enhanced further when it is employed in conjunction with a cutting edge design optimization scheme such as the surrogate based multi-objective, multi-fidelity GPU accelerated schemes employed above. Prometheus enables such studies to be carried out with ease but these advanced optimization schemes enable results to be obtained faster.

\section{Acknowledgements}

The support of both ANSYS and Loughborough University in carrying out the presented work is greatly acknowledged.

\section{References}

[1] Painchaud-Ouellet, S., Tribes, C., Trépanier, J., and Pelletier, D., 2006. "Airfoil shaped optimization using a nonuniform rational B-spline parameterization under thickness constraint”. AIAA Journal, 44(10), pp. 21702178.

[2] Shahrokhi, A., and Jahangirian, A., 2007. "Airfoil shape parameterization for optimum navier-stokes de- sign with genetic algorithm”. Aerospace Science and Technology, 11(6), pp. 443-450.

[3] Brooks, C., Forrester, A., Keane, A., and Shahpar, S., 2011. "Multi-fidelity design optimisation of a transonic compressor rotor". In 9th European Turbomachinery Conference, Istanbul, Turkey, 21st-25th March.

[4] Epstein, B., Jameson, A., Peigin, S., Roman, D., Harrison, N., and Vassberg, J., 2009. "Comparative study of three-dimensional wing drag minimization by different optimization techniques". Journal of Aircraft, 46(2), pp. 526-541.

[5] Peigin, S., and Epstein, B., 2007. "Efficient approach for multipoint aerodynamic wing design of business jet aircraft". AIAA Journal, 45(11), pp. 2612-2621.

[6] Despierre, A., Stuttaford, P., and Rubini, P., 1997. "Preliminary gas turbine combustor design using a genetic algorithm". In In Int. Gas Turbine and Aeroengine Congress \& Exhibition, 2-5th June, Orlando, Florida, ASME 97-GT-72.

[7] Rogero, J., and Rubini, P., 2001. "Optimisation of combustor wall heat transfer and pollutant emmissions for preliminary design using evolutionary techniques". In Proceedings of ISOABE, 15th International Symposium on Airbreathing Engines, 2-7 September, Bangalore, India.

[8] Duchaine, F., Morel, T., and Gicquel, M., 2009. "Computational-fluid-dynamics-based kriging optimization tool for aeronautical combustion chambers". AIAA Journal, 47(3), pp. 631-645.

[9] Voutchkov, I., Keane, A., and Fox, R., 2006. "Robust structural design of a simplified jet engine model using multiobjective optimization”. In 11th AIAA/ISSMO Multidisciplinary Analysis and Optimization Conference, Portsmouth, Virginia, USA, September 6-8.

[10] Toal, D., Keane, A., Benito, D., Dixon, J., Yang, J., Price, M., Robinson, T., Remouchamps, A., and Kill, N., 2014. "Multi-fidelity multidisciplinary whole engine thermo-mechanical design optimization". Journal of Propulsion and Power, 30(6), pp. 1654-1666.

[11] Zhang, X., Toal, D., Bressloff, N., Keane, A., Witham, F., Gregory, J., Stow, S., Goddard, C., Zedda, M., and Rodgers, M., 2014. "Prometheus: a geometry-centric optimisation system for combustor design". In ASME Turbo Expo 2014: Turbine Technical Conference and Exposition, Dusseldorf, DE.

[12] Stuttaford, P., and Rubini, P., 1997. "Preliminary gas turbine combustor design using a network approach". Journal of Engineering for Gas Turbines and Power, 119(3), pp. 546-552.

[13] Simpson, T., Peplinski, J., Kock, P., and Allen, J., 2001. "Metamodels for computer-based engineering design: Survey and recommendations". Engineering with Computers, 17(2), pp. 129-150.

[14] Queipo, N., Haftka, R., Shyy, W., Goel, T., Vaidyanathan, R., and Tucker, P., 2005. "Surrogatebased analysis and optimization". Progress in Aerospace Sciences, 41, pp. 1-28.

[15] Forrester, A., and Keane, A., 2009. "Recent advances in 
surrogate-based optimization". Progress in Aerospace Sciences, 45(1-3), pp. 50-79.

[16] Sacks, J., Welch, W., Mitchell, T., and Wynn, H., 1989. "Design and analysis of computer experiments". Statistical Science, 4(4), pp. 409-435.

[17] Jones, D., 2001. "A taxonomy of global optimization methods based on response surfaces". Journal of Global Optimization, 21(4), pp. 345-383.

[18] Jones, D., Schonlau, M., and Welch, W., 1998. "Efficient global optimization of expensive black-box functions". Journal of Global Optimization, 13(4), pp. 455492.

[19] Toal, D., Forrester, A., Bressloff, N., Keane, A., and Holden, C., 2009. "An adjoint for likelihood maximization”. Proceedings of the Royal Society A, 465(2111), pp. 3267-3287.

[20] Toal, D., Bressloff, N., Keane, A., and Holden, C., 2011. "The development of a hybridized particle swarm for kriging hyperparameter tuning". Engineering Optimization (Accepted for Publication).

[21] Forrester, A., Sóbester, A., and Keane, A., 2007. "Multi-fidelity optimization via surrogate modelling". Proceedings of the Royal Society A, 463(2088), pp. 3251-3269.

[22] Toal, D., 2015. "Some considerations regarding the use of multi-fidelity kriging in the construction of surrogate models". Structural and Multidisciplinary Optimization.

[23] Kuya, Y., Takeda, K., Zhang, X., and Forrester, A., 2011. "Multifidelity surrogate modeling of experimental and computational aerodynamic data sets". AIAA Journal, 49(2), pp. 289-298.

[24] Toal, D., and Keane, A., 2011. "Efficient multipoint aerodynamic design optimization via co-kriging". Journal of Aircraft, 48(5), pp. 1685-1695.

[25] Yamazaki, W., and Mavriplis, D., 2013. "derivativeenhanced variable fidelity surrogate modeling for aerodynamic functions". AIAA Journal, 51, pp. 126-137.

[26] Ghoreyshi, M., Badcock, K., and Woodgate, M., 2009. "Accelerating the numerical generation of aerodynamic models for flight simulation". Journal of Aircraft, 46(3), pp. 972-980.

[27] Han, Z., and Görtz, S., 2012. "Hierarchical kriging model for variable-fidelity surrogate modeling". AIAA Journal, 50(9), pp. 1885-1896.

[28] Han, Z., Görtz, S., and Zimmermann, R., 2013. "Improving variable-fidelity surrogate modeling via gradient-enhanced kriging and a generalised hybrid bridge function". Aerospace Science and Technology, 25(1), pp. 177-189.

[29] Han, Z., Zimmermann, R., and Görtz, S., 2012. "Alternative cokriging model for variable-fidelity surrogate modeling". AIAA Journal, 50(5), pp. 1205-1210.

[30] Kennedy, M., and O'Hagan, A., 2000. "Predicting the output from a complex computer code when fast approximations are available". Biometrika, 87(1), pp. 113.

[31] Keane, A., 2006. "Statistical improvement criteria for use in mulitobjective design optimization". AIAA Journal, 44(4), pp. 879-891.

[32] Toal, D., and Keane, A., 2012. "Non-stationary kriging for design optimization". Engineering Optimization, 44(6), pp. 741-765.

[33] Cha, C., Ireland, P., Denman, P., and Savarianandam, V., 2012. "Turbulence level are high at the combustorturbine interface". In Proceedings of the ASME Turbo Expo 2012, Copenhagen, Denmark.

[34] Deb, K., 2001. Multi-Objective Optimization using Evolutionary Algorithms. Wiley. 